\title{
Structural evolution, strain and elasticity of perovskites at high pressures and temperatures
}

\author{
Michael A. CARPENTER*, Peter SondergelD*, Baosheng Li**, Robert C. LIEBERMANN**, \\ Joanna W. WALSH*, Jürgen SCHREUER ${ }^{* * *}$ and Timothy W. DARLING ${ }^{* * *}$ \\ *Department of Earth Sciences, Downing Street, Cambridge CB2 3EQ, U.K. \\ ${ }^{* *}$ Department of Geosciences, SUNY at Stony Brook, Stony Brook, New York 11794, U.S.A. \\ *** Institut für Mineralogie/Kristallographie, Universität Frankfurt, Senckenberganlage 30, \\ D-60054, Frankfurt am Main, Germany \\ ***** Department of Physics, University of Nevada, Reno, NV 89557, U.S.A.
}

\begin{abstract}
Octahedral tilting transitions in perovskites are usually identified by the significant lattice distortions which accompany them. The underlying mechanism of coupling between the tilts and the macroscopic strain also gives rise to large anomalies in single crystal and bulk elastic moduli. Landau theory provides an effective framework for describing these different changes in properties and relating them, quantitatively, to the evolution of the driving order parameter for the transition. This approach has been used to analyse the overall elastic behaviour of perovskites belonging to the $\mathrm{CaTiO}_{3}-\mathrm{SrTiO}_{3}$ (CST) solid solution, which is expected to be closely analogous to the behaviour of silicate perovskites at higher pressures and temperatures. $P m \overline{3} m \leftrightarrow I 4 / \mathrm{mcm}$ and $I 4 / m c m \leftrightarrow P n m a$ transitions in CST perovskites are marked by changes in the shear modulus of $\sim 10-30 \%$. The evolution of the order parameter and, hence, of the octahedral tilt angles through these can be followed through the variations of spontaneous strains extracted from high resolution lattice parameter data. Contributions to the elastic softening which are due to strain/octahedral tilt coupling have been calculated using a fully parameterised Landau model of the $P m \overline{3} m \leftrightarrow I 4 / \mathrm{mcm}$ transition as a function of temperature, pressure and composition. Differences between calculated elastic constants and experimental data from Dynamical Mechanical Analysis, pulse-echo ultrasonics and Resonant Ultrasound Spectroscopy suggest that a proportion of the total softening in tetragonal samples may be due to anelastic effects. The anelastic contributions are observed at frequencies of both a few $\mathrm{Hz}$ and 10's of $\mathrm{MHz}$, and can be understood in terms of strain contributions arising from movements of transformation twin walls in response to an externally applied shear stress. Similar transitions in other perovskites are likely to display small anomalies in the bulk modulus, due to weak coupling between octahedral tilts and volume strain, but much larger anomalies in the shear modulus. The elastic properties of tetragonal and orthorhombic structures are likely to be quite different due to different anelastic contributions from twin wall displacements.
\end{abstract}

Keywords: Phase transitions, Elastic constants, Landau theory, Anelasticity, $\mathrm{CaTiO}_{3}-\mathrm{SrTiO}_{3}$ solid solution

\section{INTRODUCTION}

Of all the physical properties of minerals which are investigated in an earth sciences context, probably those which have the most widespread and pervasive influence are the elastic constants. The importance of seismics in geophysics is self-evident, but many geochemical processes are also directly or indirectly dependent on elastic properties. For example, partitioning of trace elements between crystals and liquids appears to depend systematically on the

M.A. Carpenter,mc43@esc.cam.ac.uk Corresponding author effective elastic properties of the crystals (e.g., Van Westrenen et al., 1999), the excess thermodynamic properties of silicate solid solutions can be understood in terms of purely elastic effects (e.g., Carpenter and Boffa Ballaran, 2001; Boffa Ballaran and Carpenter, 2003; Tarantino et al., 2003), and the surfaces of crystals differ from their interiors substantially as a consequence of elastic relaxations (Conti and Salje, 2001). For the most part, the elastic properties of minerals vary smoothly with changing pressure, temperature and composition, permitting more or less safe extrapolations or interpolations beyond or between sets of experimental data. If, on the other 


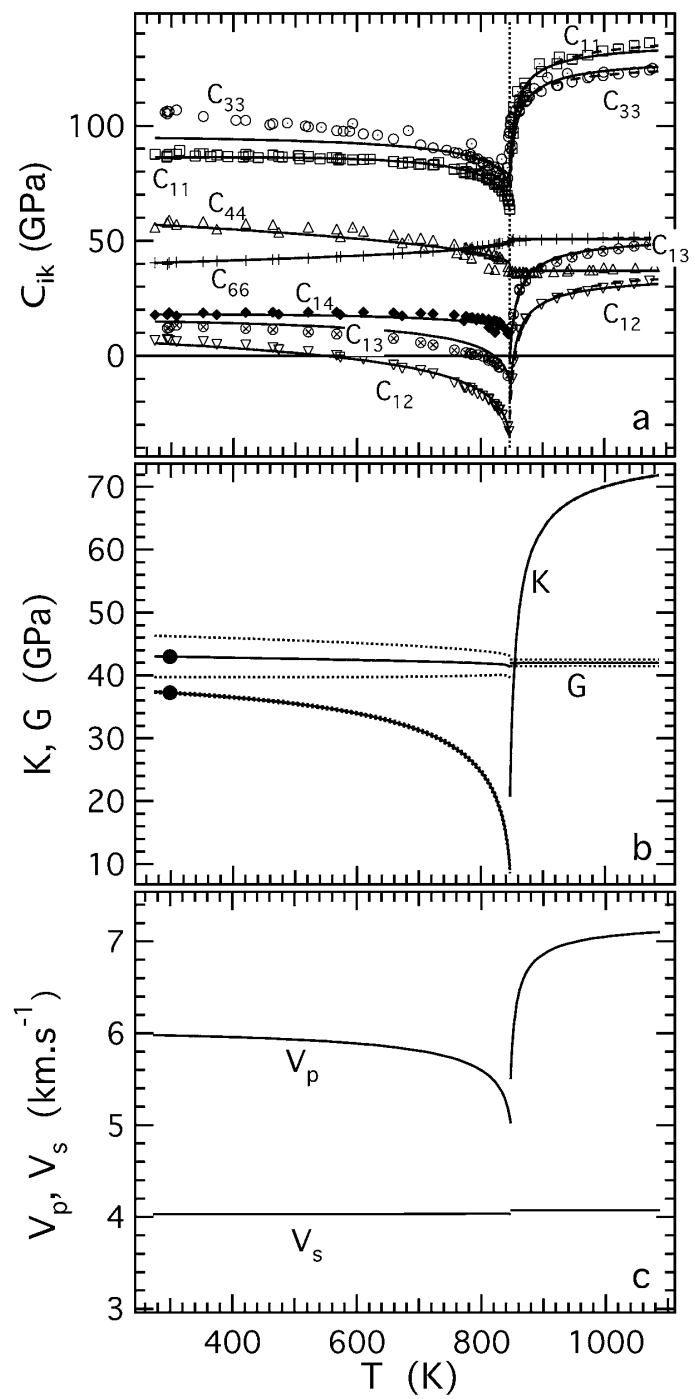

Figure 1. Changes in elastic properties through the $\alpha \leftrightarrow \beta$ transition as a function of temperature in quartz (after Carpenter et al., 1998b; Carpenter, 2006). (a) Single crystal elastic constants from the literature (individual symbols) are described by solutions to a Landau free energy expansion for $\alpha$-quartz and by an expression for the effects of fluctuations in $\beta$-quartz. (b) Variations of the bulk $(K)$ and shear $(G)$ moduli for a polycrystalline sample based on the calculated variations of individual elastic constants shown in (a). Solid lines are averages of the Voigt and Reuss limits, which are shown as dotted lines. Solid circles are bulk and shear moduli obtained for a polycrystalline quartz sample by Resonant Ultrasound Spectroscopy at ambient conditions (M.A. Carpenter and T.W. Darling, unpublished data). (c) Variation of $\mathrm{P}-$ and $\mathrm{S}^{-}$ wave velocities, $V_{\mathrm{p}}$ and $V_{\mathrm{s}}$, calculated using the Voigt/Reuss averages shown in (b).

hand, a mineral undergoes a displacive phase transition, its elastic constants can change abruptly by 10 's of percent. The softening or stiffening can also extend to many hundreds of ${ }^{\circ} \mathrm{C}$ or tens of $\mathrm{GPa}$ away from a transition point, as illustrated in Figures 1-3 for transitions in quartz, stishovite and spodumene. The main objective of the present paper is to give an overview of recent work on the variations in strain and elasticity that accompany octahedral tilting transitions in perovskites. This includes references to new theory and experimental results which will be published in full, elsewhere. The system $\mathrm{CaTiO}_{3}{ }^{-}$ $\mathrm{SrTiO}_{3}$ was chosen as an analogue for silicate perovskites, and an overall approach based on Landau theory is used to describe anomalies observed by a variety of experimental methods. It is worth noting that interest in the geophysical or geochemical properties of silicate perovskites represents only a small fraction of the total scientific interest in the solid state properties of man-made perovskites, such as are used in ferroic devices, for example.

Investigations of the layered structure of the earth tend to be focused on discontinuities in elastic behaviour because these are usually more revealing of temperature $(T)$ and composition $(X)$ conditions than the smooth variations with increasing pressure $(P)$ of apparently homogeneous regions. In the case of the reconstructive transition from olivine to wadsleyite, changes in density and seismic velocities across a narrow temperature interval are correlated with PTX information from experimental phase diagrams to provide constraints on $T$ and $X$ at a given depth (e.g., Agee, 1998). Similar information could be provided by discontinuities in the elastic properties of individual minerals, though these may have a distinct advantage in depending on relatively simple phase diagrams. For example, if the transformation from $\beta$-quartz (hexagonal) to $\alpha$-quartz (trigonal) occurred in some silica-rich portion of the crust, it should be marked by a highly distinctive pattern of $\mathrm{P}$ - and $\mathrm{S}$-wave velocities - namely a large change in $V_{\mathrm{p}}$ and almost no change in $V_{\mathrm{s}}$ (Fig. 1; Kern 1979, 1982; Carpenter, 2006). The $P T$ trajectory of this transition is well constrained and Mechie et al. (2004) have used it to determine temperature at a depth of $18 \mathrm{~km}$ in the Tibetan crust. Marini and Manzella (2005) interpreted a reflecting layer within the lithosphere of Southern Tuscany on a similar basis. The $P 4_{2} / \mathrm{mnm} \leftrightarrow$ Pnnm transition at high pressures in stishovite, $\mathrm{SiO}_{2}$, is also expected to cause large changes in elastic properties, though the total softening will be spread out over a wider interval owing to the pseudo-proper ferroelastic character. A large anomaly in the shear modulus would be identified through anomalies in both $V_{\mathrm{p}}$ and $V_{\mathrm{s}}$ (Fig. 2; Carpenter et al., 2000; Hemley et al., 2000). Hirose et al. (2005) have explained seismic features with similar attributes in terms of this transition occurring in stishovite at $\sim 1500 \mathrm{~km}$ depth in subducted MORB crust, northeast of the Mariana subduction zone. A phase transition in monoclinic pyroxene analogous to the $C 2 / c \leftrightarrow P 2_{1} / c$ transition in spodumene, $\mathrm{LiAlSi}_{2} \mathrm{O}_{6}$, would produce yet another distinctive pattern and potential geophysical marker. In spod- 


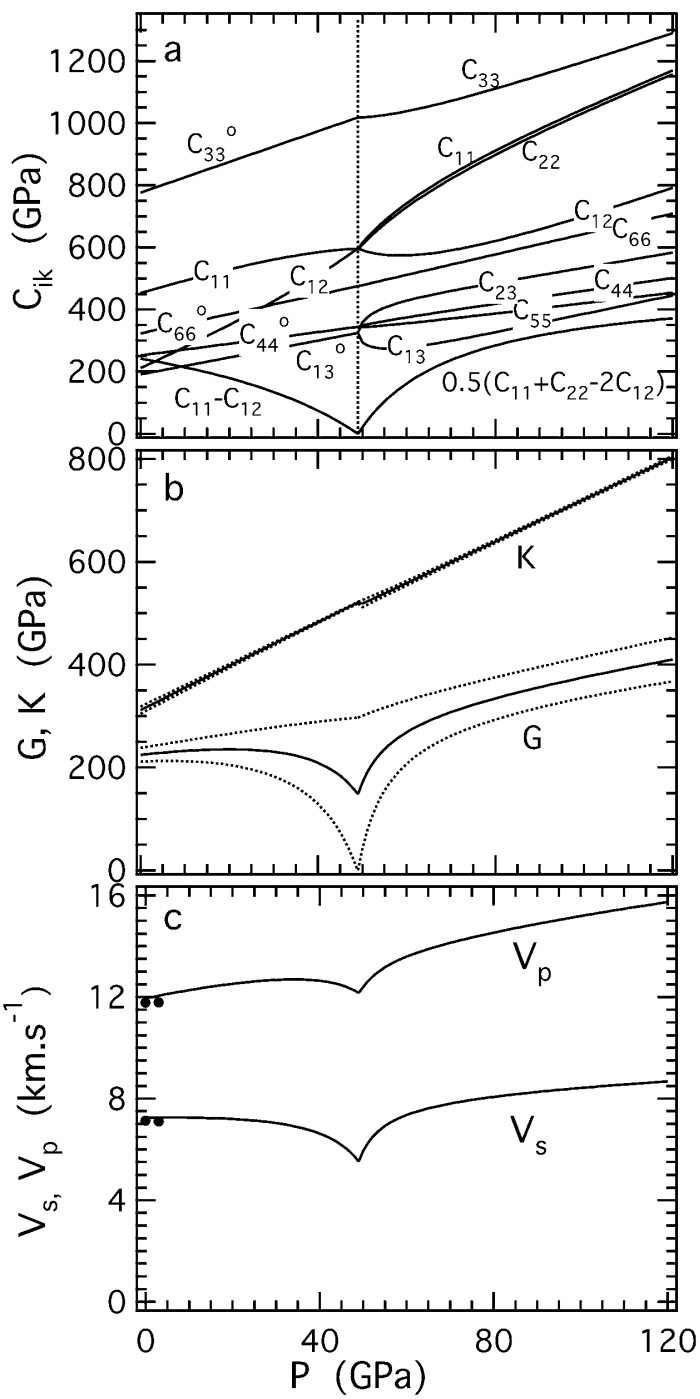

Figure 2. Elastic properties of stishovite as a function of pressure through the tetragonal $\leftrightarrow$ orthorhombic transition at $\sim 50 \mathrm{GPa}$ (after Carpenter et al., 2000; Hemley et al., 2000; Carpenter, 2006). (a) Single crystal elastic constants calculated using a Landau model. The superscript ${ }^{\circ}$ identifies elastic constants of the tetragonal structure without the influence of the phase transition. (b) Bulk and shear moduli calculated as the average of Voigt and Reuss limits for a polycrystalline sample. Dotted lines represent the Voigt and Reuss limits. (c) $\mathrm{P}^{-}$and $\mathrm{S}$-wave velocities through a polycrystalline sample, calculated using the Voigt/Reuss averages shown in (b). Filled circles are experimental values obtained by $\mathrm{Li}$ et al. (1996) at room pressure and in-situ at $3 \mathrm{GPa}$.

umene this transition has first order character and gives sharp discontinuities in compressional wave velocities for selected single crystal orientations (Sondergeld et al., 2006). A semi-quantitative Landau model predicts discontinuities in both the bulk modulus, $K$, and the shear modulus, $G$, and, hence, in both $V_{\mathrm{p}}$ and $V_{\mathrm{s}}$ for polycrystalline samples (Fig. 3). The effects of such phase transitions need not be restricted solely to variations of the elastic

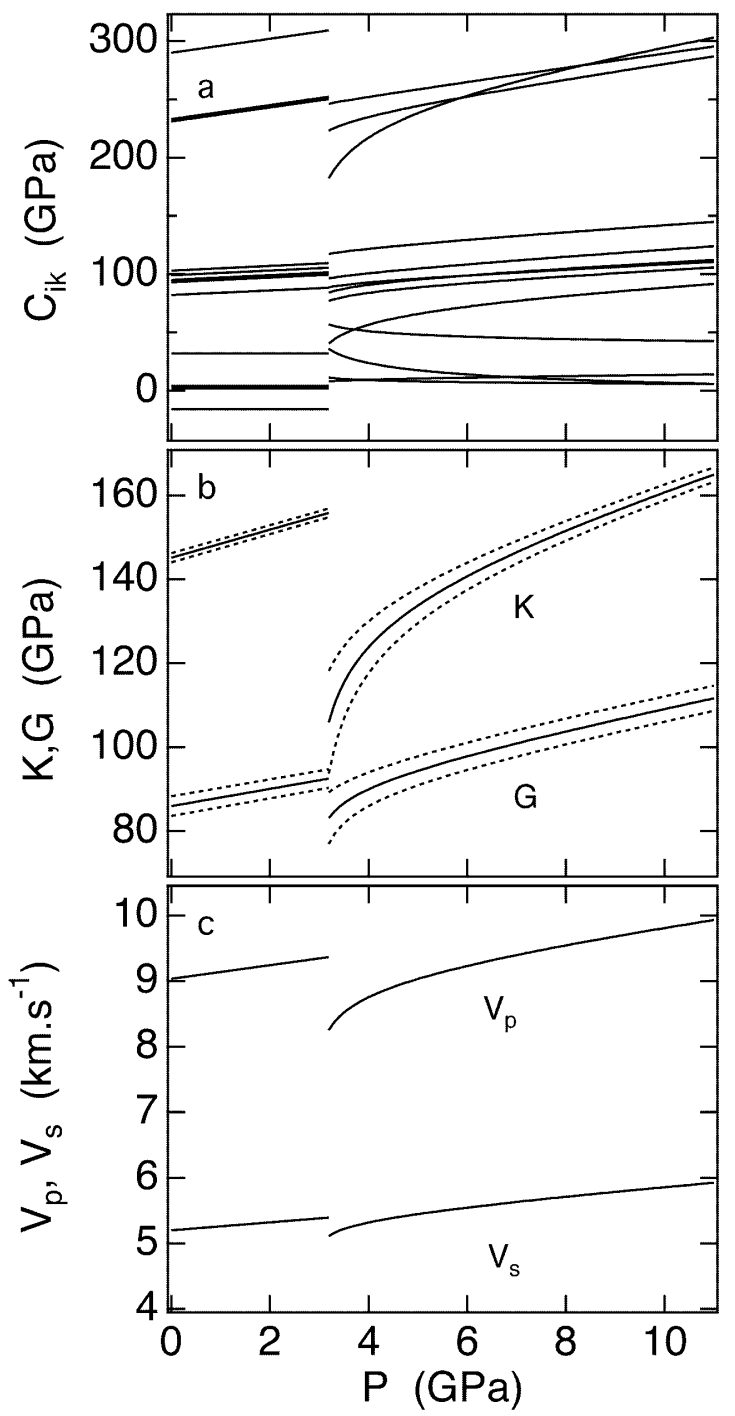

Figure 3. Variations of the elastic properties of spodumene, as calculated using a Landau solution for the first order $C 2 / c \leftrightarrow P 2{ }_{1} / c$ transition at $\sim 3.2 \mathrm{GPa}$ (after Sondergeld et al., 2006). The $P 2_{1} / c$ structure is stable above the transition point. (a) Individual elastic constants. For the benefit of clarity, in terms of the overall pattern of elastic constant variations, labels for $C_{11}, C_{22}, C_{12}$, etc. are not shown. Note that a sharp jump is expected in all elastic constants at the transition point and that this pattern is quite different from the patterns of elastic constant variations expected for quartz (Fig. 1) and stishovite (Fig. 2). (b) Bulk and shear moduli calculated for a polycrystalline sample using the single crystal elastic constants shown in (a). Solid lines are averages of the Voigt and Reuss limits, which are shown as dotted lines. (c) Variations of $\mathrm{P}-$ and $\mathrm{S}$-wave velocities calculated using the Voigt/Reuss average values of bulk and shear moduli shown in (b).

constants themselves. For example, Cordier et al. (2004) have shown that elastic anisotropy caused by the phase transition in stishovite would contribute to the development of preferred orientations in polycrystalline samples subjected to non-hydrostatic stress. Texturing of this type would give rise to a strongly anisotropic seismic signa- 
ture.

Perovskites show a greater diversity of phase transitions than any other man-made or natural materials. The orthorhombic (Pnma) structure of $(\mathrm{Mg}, \mathrm{Fe}) \mathrm{SiO}_{3}$ and the tetragonal $(I 4 / \mathrm{mcm})$ structure of $\mathrm{CaSiO}_{3}$ could both develop by octahedral tilting transitions from their holosymmetric $(P m \overline{3} m)$ forms, for example. If $P m \overline{3} m \leftrightarrow I 4 / m c m$ or $I 4 / \mathrm{mcm} \leftrightarrow P n m a$ transitions occurred in these within the mantle, significant elastic anomalies, analogous to those shown in Figures 1-3, would arise. The origin of this anomalous softening need not be restricted to the direct influence of the transitions themselves, however. It was recognised from the early days of elastic constant measurements on $\mathrm{SrTiO}_{3}$ that the displacement of transformation twin walls in response to an applied shear stress can give rise to additional softening and attenuation effects (Rehwald, 1971; Fossheim and Berre, 1972). These can be large in magnitude, giving rise to so-called superelasticity (Schranz et al., 1999; Kityk et al., 2000a, 2000b; Binder and Knorr, 2001; Lemanov et al., 2002; Harrison and Redfern, 2002; Harrison et al., 2003, 2004a, 2004b, 2004c). Equivalent behaviour in silicate perovskites cannot be investigated directly because of the high confining pressure required to stabilise them, but a useful analogue system is the binary solid solution $\mathrm{CaTiO}_{3}{ }^{-}$ $\mathrm{SrTiO}_{3}$ (CST). This system displays all the relevant strain, transformation and elasticity phenomena at pressures and temperatures which are readily accessible to in-situ measurements.

\section{PHASE TRANSITIONS IN (Ca,Sr) $\mathrm{TiO}_{3}$ PEROVSKITES}

Transition temperatures for the $P m \overline{3} m \leftrightarrow I 4 / m c m$ and $I 4 / \mathrm{mcm} \leftrightarrow P$ Pma transitions in CST perovskites vary systematically with composition (Fig. 4a). These are quite well constrained for temperatures above room temperature. Structural behaviour at lower temperatures in samples with compositions at the $\mathrm{Sr}$-rich end of the solid solution is more controversial, but is not relevant in the present context. Transition pressures are less well constrained, but a possible $P X$ phase diagram for room temperature is shown in Figure 4b. The transitions occur by cooperative tilting of the $\mathrm{TiO}_{6}$ octahedra, and are associated with $\mathrm{M}$ and $\mathrm{R}$ special points of the Brillouin zone. All such tilting transitions can be described formally using a Landau free energy expansion in which there are three order parameter components, $q_{1}{ }^{-} q_{3}$ and $q_{4}{ }^{-} q_{6}$, associated with each special point (Howard and Stokes, 1998; Carpenter et al., 2001):
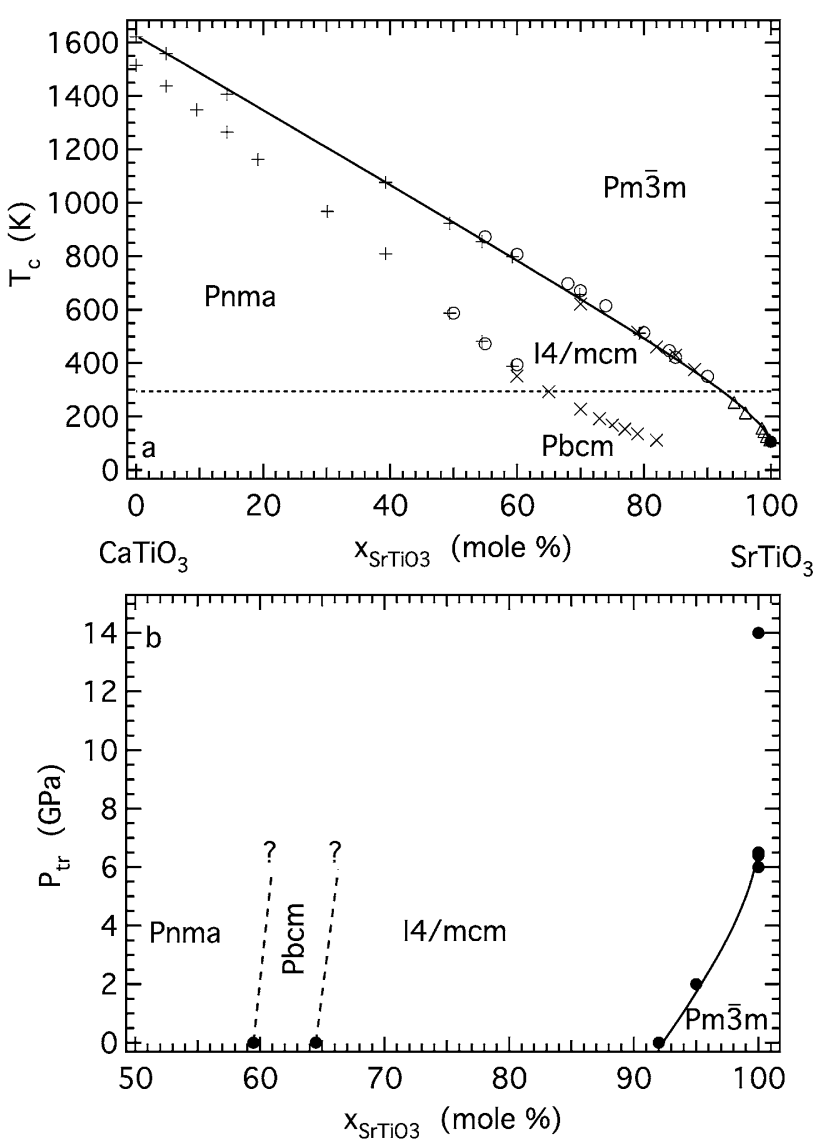

Figure 4. $T-X$ and $P-X$ phase diagrams for CST perovskites (M.A. Carpenter, unpublished data). (a) Summary of data from the literature for transition temperatures in the binary solid solution $\mathrm{CaTiO}_{3}-\mathrm{SrTiO}_{3}$, including a stability field for the $\mathrm{Pbcm}$ structure (Lemanov, 1997; Ranjan and Pandey, 2001; Qin et al., 2000; Harrison et al., 2003). The horizontal broken line represents room temperature. (Low temperature transitions at $\mathrm{Sr}^{-}$-rich compositions are not included). (b) Transition pressures as a function of composition at room temperature. Approximate composition limits for the $\mathrm{Pbcm}$ structure at ambient conditions are from Mishra et al. (2006). Data for the $P m \overline{3} m \leftrightarrow I 4 / \mathrm{mcm}$ transition in $\mathrm{SrTiO}_{3}$ are from Ishidate and Isonuma (1992), Bonello et al. (1989a, 1989b) and Grzechnik et al. (1997). The solid curve is the calculated transition pressure based on Equation 14 and the variation of $T_{\mathrm{c}}$ shown in (a). The data point for CST95 is from M.A. Carpenter (unpublished data). The possible tetragonal $\leftrightarrow$ orthorhombic transition reported by Grzechnik et al. (1997) to be between $\sim 12$ and $\sim 15 \mathrm{GPa}$ is shown arbitrarily at $14 \mathrm{GPa}$. Broken lines are arbitrary guesses at the trends for $I 4 / \mathrm{mcm} \leftrightarrow P b \mathrm{~cm}$ and $P b c m \leftrightarrow P n m a$ transitions. [Yamanaka et al. (2002) reported that CST65 has Pnma symmetry at $3.5 \mathrm{GPa}]$.

$$
\begin{aligned}
G & =\frac{1}{2} a_{1} \Theta_{\mathrm{s} 1}\left(\operatorname{coth}\left(\frac{\Theta_{\mathrm{s} 1}}{T}\right)-\operatorname{coth}\left(\frac{\Theta_{\mathrm{s} 1}}{T_{\mathrm{c} 1}}\right)\right)\left(q_{1}^{2}+q_{2}^{2}+q_{3}^{2}\right) \\
& +\frac{1}{2} a_{2} \Theta_{\mathrm{s} 2}\left(\operatorname{coth}\left(\frac{\Theta_{\mathrm{s} 2}}{T}\right)-\operatorname{coth}\left(\frac{\Theta_{\mathrm{s} 2}}{T_{\mathrm{c} 2}}\right)\right)\left(q_{4}^{2}+q_{5}^{2}+q_{6}^{2}\right)
\end{aligned}
$$




$$
\begin{aligned}
& +\frac{1}{4} b_{1}\left(q_{1}^{2}+q_{2}^{2}+q_{3}^{2}\right)^{2}+\frac{1}{4} b_{1}\left(q_{1}^{4}+q_{2}^{4}+q_{3}^{4}\right) \\
& +\frac{1}{4} b_{2}\left(q_{4}^{2}+q_{5}^{2}+q_{6}^{2}\right)^{2}+\frac{1}{4} b_{2}^{\prime}\left(q_{4}^{4}+q_{5}^{4}+q_{6}^{4}\right) \\
& +\frac{1}{6} c_{1}\left(q_{1}^{2}+q_{2}^{2}+q_{3}^{2}\right)^{3}+\frac{1}{6} c_{1}\left(q_{1} q_{2} q_{3}\right)^{2} \\
& +\frac{1}{6} c_{1}^{\prime \prime}\left(q_{1}^{2}+q_{2}^{2}+q_{3}^{2}\right)\left(q_{1}^{4}+q_{2}^{4}+q_{3}^{4}\right)+\frac{1}{6} c_{2}\left(q_{4}^{2}+q_{5}^{2}+q_{6}^{2}\right)^{3} \\
& +\frac{1}{6} c_{2}\left(q_{4} q_{5} q_{6}\right)^{2}+\frac{1}{6} c_{2}^{\prime \prime}\left(q_{4}^{2}+q_{5}^{2}+q_{6}^{2}\right)\left(q_{4}^{4}+q_{5}^{4}+q_{6}^{4}\right) \\
& +\lambda_{\mathrm{q}}\left(q_{1}^{2}+q_{2}^{2}+q_{3}^{2}\right)\left(q_{4}^{2}+q_{5}^{2}+q_{6}^{2}\right) \\
& +\lambda_{\mathrm{q}}^{\prime}\left(q_{1}^{2} q_{4}^{2}+q_{2}^{2} q_{5}^{2}+q_{3}^{2} q_{6}^{2}\right)+\lambda_{1} e_{\mathrm{a}}\left(q_{1}^{2}+q_{2}^{2}+q_{3}^{2}\right) \\
& +\lambda_{2} e_{\mathrm{a}}\left(q_{4}^{2}+q_{5}^{2}+q_{6}^{2}\right) \\
& +\lambda_{3}\left[\sqrt{3} e_{0}\left(q_{2}^{2}-q_{3}^{2}\right)+e_{\mathrm{t}}\left(2 q_{1}^{2}-q_{2}^{2}-q_{3}^{2}\right)\right] \\
& +\lambda_{4}\left[\sqrt{3} e_{\mathrm{o}}\left(q_{5}^{2}-q_{6}^{2}\right)+e_{\mathrm{t}}\left(2 q_{4}^{2}-q_{5}^{2}-q_{6}^{2}\right)\right] \\
& +\lambda_{5}\left(e_{4} q_{4} q_{6}+e_{5} q_{4} q_{5}+e_{6} q_{5} q_{6}\right) \\
& +\lambda_{6}\left(q_{1}^{2}+q_{2}^{2}+q_{3}^{2}\right)\left(e_{4}^{2}+e_{5}^{2}+e_{6}^{2}\right)+\lambda_{7}\left(q_{1}^{2} e_{6}^{2}+q_{2}^{2} e_{4}^{2}+q_{3}^{2} e_{5}^{2}\right) \\
& +\frac{1}{4}\left(C_{11}^{o}-C_{12}^{o}\right)\left(e_{\mathrm{o}}^{2}+e_{\mathrm{t}}^{2}\right) \\
& +\frac{1}{6}\left(C_{11}^{\mathrm{o}}+2 C_{12}^{\mathrm{o}}\right) e_{\mathrm{a}}^{2}+\frac{1}{2} C_{44}^{\mathrm{o}}\left(e_{4}^{2}+e_{5}^{2}+e_{6}^{2}\right) \text {. }
\end{aligned}
$$

Note that, here, the symbol $G$ refers to the excess free energy due to a phase transition, rather than the shear modulus. The $I 4 / \mathrm{mcm}$ structure has $q_{4} \neq 0, q_{1}=q_{2}=q_{3}=$ $q_{5}=q_{6}=0$, and the Pnma structure has $q_{2} \neq 0, q_{4}=q_{6} \neq 0$, $q_{1}=q_{3}=q_{5}=0 . T_{\mathrm{c} 1}$ and $T_{\mathrm{c} 2}$ are critical temperatures for the $\mathrm{M}$ and $\mathrm{R}$ point instabilities, $a_{1}, a_{2}, b_{1}, b_{2}, \ldots$ are standard Landau coefficients, $\Theta_{\mathrm{s} 1}$ and $\Theta_{\mathrm{s} 2}$ are saturation temperatures, $\lambda_{1}, \lambda_{2}, \ldots$ are coupling coefficients, $e_{\mathrm{a}}, e_{\mathrm{o}}, e_{\mathrm{t}}$ are symmetry-adapted strains and $C_{i k}^{0}$ are elastic constants of the parent cubic structure. In most cases of tilting transitions in perovskites the structural parameter which should effectively behave as the order parameter is the rotation angle, $\phi$, of the octahedra (Glazer, 1972, 1975; Woodward, 1997; Howard and Stokes, 1998). This is zero in the cubic phase and non-zero in the derivative structures. Each of $q_{1}, q_{2}, \ldots$ can then be related to angles of rotation about different tilt axes. Details of Landau theory applied to minerals have been described in many reviews, including, for example, Salje (1991, 1992), Carpenter (1988, 1992, 2000, 2002), Carpenter et al. (1998a), Carpenter and Salje (1998), Redfern (2000) and Salje et al. (2005). Selected results for perovskites are given below without further
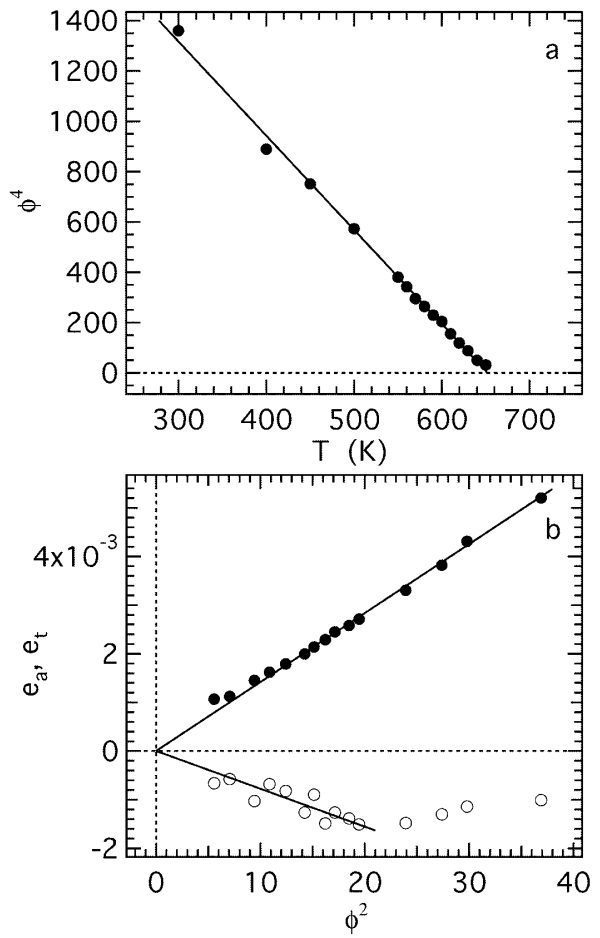

Figure 5. Octahedral tilt angle, $\phi$ (in degrees), and spontaneous strains through the $P m \overline{3} m \leftrightarrow I 4 / m c m$ transition in CST70. (a) The tilt angle for CST70 varies as $\phi^{4} \propto\left(T_{\mathrm{c}}-T\right)$, implying that the transition is tricritical (after Howard et al., 2005). (b) The tetragonal strain ( $e_{\mathrm{t}}$, filled circles), calculated from lattice parameter data of Howard et al. (2005), varies linearly with $\phi^{2}$. The volume strain $\left(e_{\mathrm{a}}\right.$, open circles) is subject to larger uncertainty than $e_{\mathrm{t}}$, but varies linearly with $\phi^{2}$ only over a limited range.

explanation.

\section{STRUCTURAL EVOLUTION, OCTAHEDRAL TILTING AND SPONTANEOUS STRAIN}

Simple rotation of $\mathrm{TiO}_{6}$ octahedra about the [001] axis in CST perovskites, with alternate layers parallel to the (001) plane having rotations of opposite sign, leads to the I4/mcm structure. For $\left(\mathrm{Ca}_{0.3} \mathrm{Sr}_{0.7}\right) \mathrm{TiO}_{3}$ (CST70), the tilt angle varies with temperature as $\phi^{4} \propto\left(T_{\mathrm{c}}-T\right)$, where $T_{\mathrm{c}}=$ $652 \mathrm{~K}$ (Fig. 5a, after Howard et al., 2005). For $q_{4} \propto \phi$, this implies that the transition is tricritical in character and, hence, that the order parameter should follow a solution to Equation 1 which has

$$
q_{4}^{4}=\frac{a_{2} \Theta_{\mathrm{s} 2}}{\left(c_{2}+c_{2}^{\prime \prime}\right)}\left(\operatorname{coth}\left(\frac{\Theta_{\mathrm{s} 2}}{T_{\mathrm{c} 2}}\right)-\operatorname{coth}\left(\frac{\Theta_{\mathrm{s} 2}}{T}\right)\right) .
$$

Accompanying the tilting is a tetragonal distortion of the unit cell, $e_{\mathrm{t}}$, and a volume strain, $e_{\mathrm{a}}$. In terms of linear strains $e_{1}, e_{2}, e_{3}$, these symmetry-adapted strains are given by 


$$
\begin{aligned}
& e_{\mathrm{t}}=\frac{1}{\sqrt{3}}\left(2 e_{3}-e_{1}-e_{2}\right) \\
& e_{\mathrm{a}}=\left(e_{1}+e_{2}+e_{3}\right)
\end{aligned}
$$

where $e_{1}=\left(a-a_{\mathrm{o}}\right) / a_{\mathrm{o}}, e_{2}=\left(b-a_{\mathrm{o}}\right) / a_{\mathrm{o}}, e_{3}=\left(c-a_{\mathrm{o}}\right) / a_{\mathrm{o}} ; a, b$, $c$ are lattice parameters of the tetragonal structure at a given temperature and $a_{\mathrm{o}}$ is the lattice parameter of the cubic structure extrapolated to the same temperature. Setting the equilibrium condition $\partial G / \partial e=0$ leads to the relationships

$$
\begin{aligned}
& e_{\mathrm{t}}=-\frac{2 \lambda_{4} q_{4}^{2}}{\frac{1}{2}\left(C_{11}^{\mathrm{o}}-C_{12}^{\mathrm{o}}\right)} \\
& e_{\mathrm{a}}=-\frac{\lambda_{2} q_{4}^{2}}{\frac{1}{3}\left(C_{11}^{\mathrm{o}}+2 C_{12}^{\mathrm{o}}\right)}
\end{aligned}
$$

and the prediction that the tetragonal and volume strains should vary linearly with $\phi^{2}$. Wherever data are available for perovskites, this appears to be true for shear strains such as $e_{\mathrm{t}}$, and the lattice parameter data of Howard et al. (2005) indeed yield the expected linear dependence (Fig. $5 b)$. Experimental uncertainties introduced in the extrapolation of $a_{\mathrm{o}}$ from high temperatures tend to result in much greater scatter for the volume strain, $e_{\mathrm{a}}$, but the non-linear behaviour for CST shown in Figure 5b is not atypical of perovskites; the same type of pattern is seen as a function of temperature in $\mathrm{CaTiO}_{3}$ and as a function of composition across the CST solid solution at room temperature (Carpenter et al., 2001). Volume strains accompanying the tilting transitions can be positive (increasing pressure favours the high symmetry form) or negative (increasing pressure favours the low symmetry form), showing that the octahedra do not behave as rigid units (Angel et al., 2005). Spontaneous strain data for the cubic $\leftrightarrow$ tetragonal transition show that it is also close to tricritical in pure $\mathrm{CaTiO}_{3}$ (Carpenter et al., 2001), but more nearly second order in $\mathrm{SrTiO}_{3}$ (Salje et al., 1998; Hayward and Salje, 1999).

\section{ELASTIC ANOMALIES AS A FUNCTION OF TEMPERATURE}

Structural phase transitions lead to elastic softening because of the coupling between spontaneous strains and the order parameter. If the order parameter relates to a particular octahedral tilt, the restoring force opposing this tilt becomes small as the transition point is approached. Because the tilts are linked to macroscopic strains via strain/order parameter coupling terms such as $\lambda e q^{2}$, a mechanism is available for the strain response to be modified by the tilting behaviour when an external shear stress is applied. Once the restoring force on the octahedra is small the crystal becomes elastically softer than it would be if the tilting instability did not exist. The important parameters are clearly the strength of coupling between the order parameter and strain, i.e. the magnitude of $\lambda$, and the shape of the energy potential which controls the tilting, i.e. $\partial^{2} G / \partial q^{2}$. Expressions for the individual elastic constants are given formally by applying

$$
C_{i k}=C_{i k}^{o}-\sum_{m} \frac{\partial^{2} G}{\partial e_{i} \partial q_{m}} \cdot\left(\frac{\partial^{2} G}{\partial q_{m}^{2}}\right)^{-1} \cdot \frac{\partial^{2} G}{\partial e_{k} \partial q_{m}}
$$

to Equation 1 (Slonczewski and Thomas, 1970). This has been widely used in relation to Landau expansions out to fourth order in $q$ (second order transitions), but only recently extended to the full sixth order potential given above (see, also, Kityk et al., 2000a). For the $P m \overline{3} m \leftrightarrow$ $I 4 / \mathrm{mcm}$ transition, individual elastic constants, such as $C_{11}$ and $C_{44}$, are expected to follow

$$
\begin{aligned}
& C_{11}=C_{22}=C_{11}^{0}-\left(2 \lambda_{2}-\frac{4}{\sqrt{3}} \lambda_{4}\right)^{2} \chi_{4} q_{4}^{2} \\
& C_{44}=C_{55}=C_{44}^{0}-\lambda_{5}^{2} \chi_{6} q_{4}^{2},
\end{aligned}
$$

where

$$
\begin{aligned}
& \chi_{4}^{-1}=\partial^{2} G / \partial q_{4}^{2}=2\left(b_{2}+b_{2}^{\prime}\right) q_{4}^{2}+4\left(c_{2}+c_{2}^{\prime \prime}\right) q_{4}^{4} \\
& \chi_{6}^{-1}=\partial^{2} G / \partial q_{6}^{2}=\left[\frac{12 \lambda_{4}^{2}}{\frac{1}{2}\left(C_{11}^{\circ}-C_{12}^{\circ}\right)}-b_{2}^{\prime}\right] q_{4}^{2}-\frac{2}{3} c_{2}^{\prime \prime} q_{4}^{4} .
\end{aligned}
$$

Bulk $(K)$ and shear $(G)$ moduli can be calculated from the individual elastic constants in the usual way by taking the average of Voigt and Reuss limits (Hill, 1952) or by using Hashin-Shtrikman bounds (Watt, 1979; Watt and Peselnik, 1980). Expressions for the Voigt limits have a form which most readily illustrates the relative importance of different parameters in determining how much softening of the bulk properties occurs. For the cubic $\leftrightarrow$ tetragonal transition these are

$$
\begin{aligned}
& K_{\mathrm{V}}=\frac{1}{3}\left(C_{11}^{\mathrm{o}}+2 C_{12}^{\mathrm{o}}\right)-4 \lambda_{2}^{2} \chi_{4} q_{4}^{2} \\
& G_{\mathrm{V}}=\frac{1}{5}\left(C_{11}^{\mathrm{o}}-C_{12}^{\mathrm{o}}+3 C_{44}^{\mathrm{o}}\right)-\frac{2}{5}\left(8 \lambda_{4}^{2} \chi_{4}+\lambda_{5}^{2} \chi_{6}\right) q_{4}^{2} .
\end{aligned}
$$




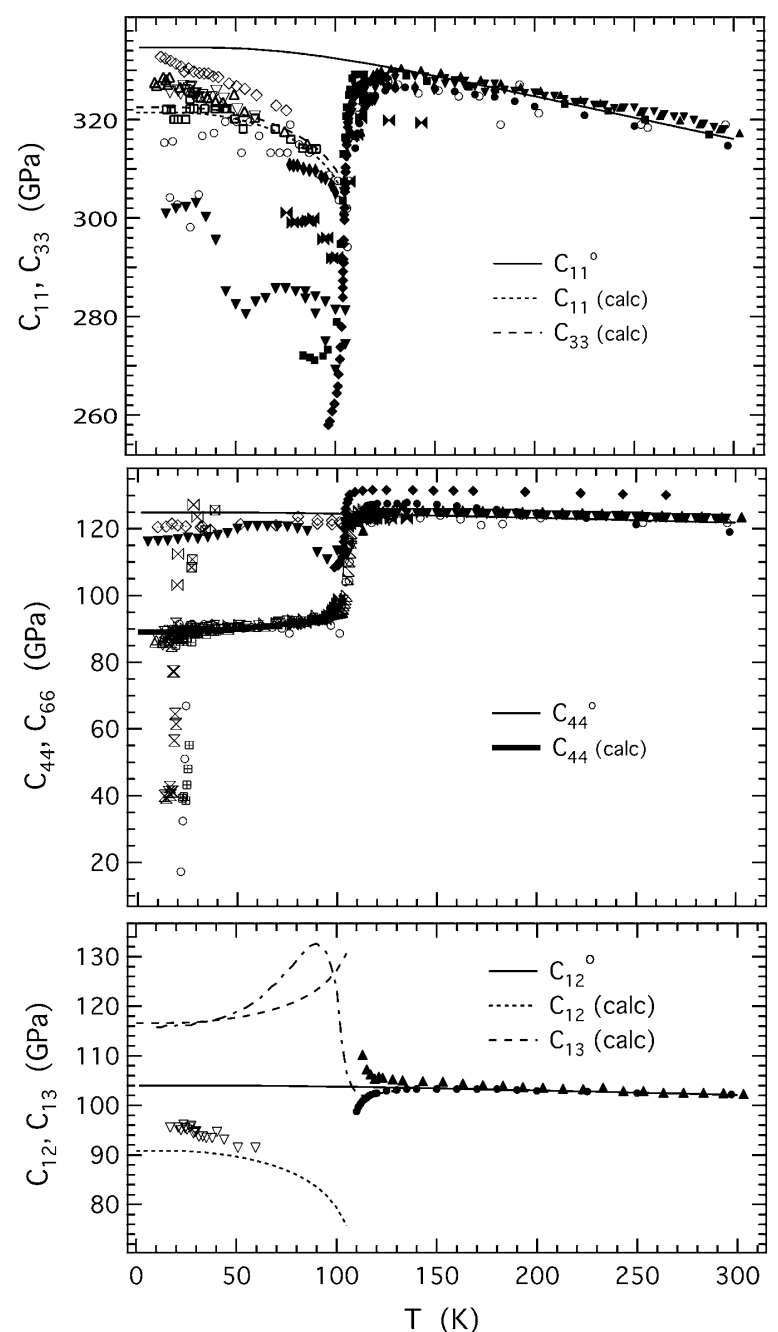

Figure 6. Comparison of calculated variations of single crystal elastic constants for $\mathrm{SrTiO}_{3}$ with a compilation of experimental data assembled from the literature (M.A. Carpenter, unpublished data). Filled symbols represent ultrasonic data; open symbols represent Brillouin data. Data for $C_{12}$ from Rehwald (1970) are shown as a continuous line (dot-dash line), as in the original paper. Calculated variations of $C_{11}, C_{33}, C_{12}, C_{13}$ and $C_{44}$ are from the Landau parameterisation. Scatter in the data for $C_{44}$ below $\sim 30 \mathrm{~K}$, as obtained from conversion of reported data for split acoustic modes directly to elastic constants, is due to additional structural changes which occur in ${ }^{18} \mathrm{O}$-enriched samples.

Calibration of all the coefficients has been completed for the $P m \overline{3} m \leftrightarrow I 4 / m c m$ transition in $\mathrm{SrTiO}_{3}$ using experimental data for heat capacity, spontaneous strain, phase relations under applied stress, etc. (M.A. Carpenter, unpublished data). This was based on the solution for the evolution of $q_{4}$ as a function of temperature obtained by Hayward and Salje (1999), in which both fourth and sixth order terms are significant (246 solution). Calculated variations of the single crystal elastic constants determined with this set of parameters are compared with a compilation of experimental data from the literature in Figure 6.

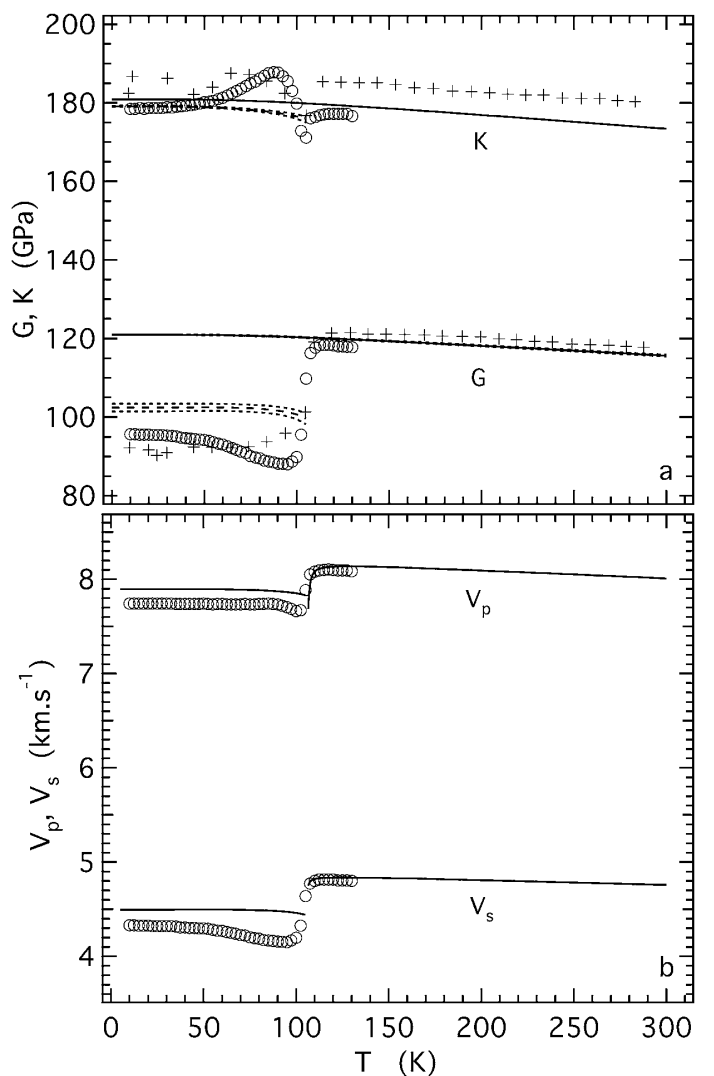

Figure 7. Elastic properties of polycrystalline $\mathrm{SrTiO}_{3}$ as a function of temperature. (a) Bulk and shear moduli calculated from single crystal elastic constants (M.A. Carpenter, unpublished data). Solid lines (cubic) and broken lines (tetragonal) are Voigt/Reuss averages based on the Landau solutions shown in Figure 6. The Voigt and Reuss limits are shown as dotted lines. Pseudocubic experimental data (i.e. from twinned crystals) of Rehwald (1970) were used to calculate values of $K$ and $G$ (average of Voigt/Reuss limits) and are shown as by open circles. Experimental data of Lei (1991) are shown as crosses. Experimental data for the bulk modulus are consistent with calculated values for tetragonal $\mathrm{SrTiO}_{3}$, but values for the shear modulus are smaller than values calculated assuming that the only softening is due to strain/order parameter coupling. (b) Variations of $\mathrm{P}$-wave and $\mathrm{S}$-wave velocities calculated using the values of $K$ and $G$ shown in (a).

Two features are immediately apparent from the comparison for $C_{11}$ and $C_{33}$. Firstly, there is close agreement between the model values for the tetragonal structure and Brillouin scattering data collected from within individual twin domains. This represents an independent test of the internal consistency of the Landau model. Secondly, pulse-echo ultrasonic data for single crystals with multiple twin domains show both a higher degree of scatter and a higher degree of softening. This supports the argument that transformation twin walls are important in causing softening which is additional to the effects of strain/order parameter coupling. Overlap of Brillouin and ultrasonic data for $C_{44}$ implies that the presence of transformation 
twins does not influence this component of the elastic constant tensor. The experimental data were themselves used to extract values for some of the Landau coefficients, however, so that agreement between the observed and calculated values is a measure of the quality of fit rather than a true test of the model. Variations of $K$ and $G$ are compared in Figure 7a, while the variations of $V_{\mathrm{p}}$ and $V_{\mathrm{s}}$ calculated from these are shown in Figure $7 \mathrm{~b}$. The phase transition would be expected to cause only a small deviation in $K$ ( $\lambda_{2}$ in Equation 12 is small), but a softening of $20 \%$ is expected in $G$. The added influence of transformation twins applies only to $G$ and a further $\sim 10 \%$ softening is obtained in measurements at frequencies of 10 's of MHz.

Softening of the elastic constants of cubic $\mathrm{SrTiO}_{3}$ ahead of the phase transition (Fig. 6) occurs by a different mechanism that is not discussed here. This type of softening is characteristic of cubic perovskites ahead of cubic $\leftrightarrow$ tetragonal or cubic $\leftrightarrow$ rhombohedral transitions (see Carpenter and Salje, 1998a, and many original references therein). A similar mechanism is responsible for softening in $\beta$-quartz ahead of the $\alpha \leftrightarrow \beta$ transition (Fig. 1).

\section{ELASTIC ANOMALIES AS A FUNCTION OF COMPOSITION}

A convenient feature of Landau theory is the ease with which variations in composition and pressure can be included simply by allowing selected parameters to become composition and pressure dependent. It is apparent from Figure $4 \mathrm{a}$ that the predominant consequence of changing Ca:Sr ratio across the CST solid solution is a systematic change in the transition temperatures. The thermodynamic character of the $P m \overline{3} m \leftrightarrow I 4 / \mathrm{mcm}$ transition also changes, however, from 246 in $\mathrm{SrTiO}_{3}$ (Salje et al., 1998; Hayward and Salje, 1999) to tricritical in CST70 (Howard et al., 2005; M.A. Carpenter, unpublished data) and CST0 (Carpenter et al., 2001). The strength of coupling to the tetragonal strain also changes, and the simplest model of the transition which accounts for these effects is one in which only two parameters are adjusted. Firstly, the transition temperature, $T_{\mathrm{c} 2}$, is allowed to vary with composition according to the experimental data. Secondly, the coupling coefficient, $\lambda_{4}$, is set at $-0.131 \mathrm{GPa}$ for the composition range CST0-CST92 but allowed to vary linearly between $-0.131 \mathrm{GPa}$ at $\mathrm{CST} 92$ and $-0.075 \mathrm{GPa}$ at CST100. All the other coefficients are assumed to remain constant. Once again, lattice parameter data can be used to test the evolution of the order parameter. At room temperature, $e_{\mathrm{t}}$ is zero in cubic CST92-CST100 but it then increases with increasing $\mathrm{Ca}^{-}$-content from CST92. The evolution of $q_{4}^{2}$ at $T=295 \mathrm{~K}$ is given by Equation 2, with

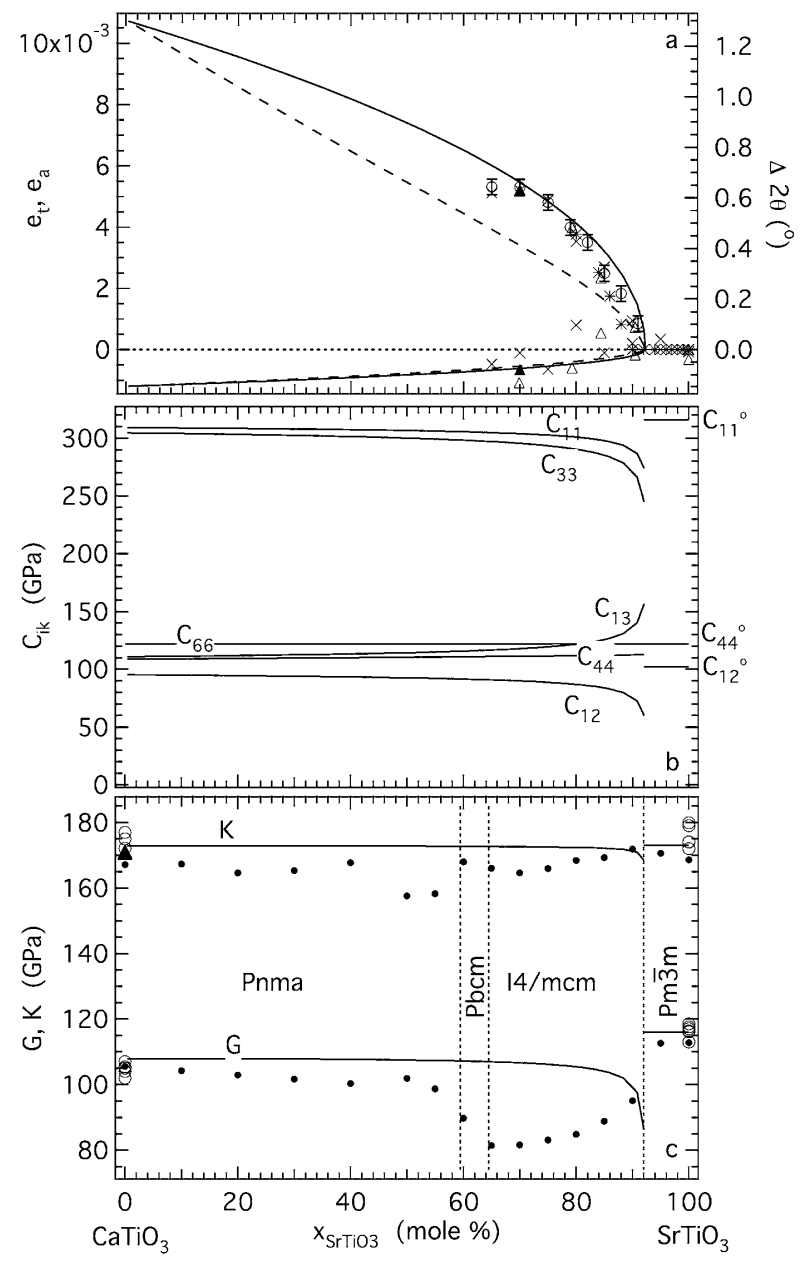

Figure 8. Strain and elasticity of CST perovskites at room temperature (from M.A. Carpenter, unpublished data). (a) Variations of the tetragonal strain, $e_{\mathrm{t}}$, and volume strains, $e_{\mathrm{a}}$, in cubic and tetragonal samples of CST, as extracted by Carpenter et al. (2001) from published lattice parameters. Open circles are experimental values from Ranjan et al. (2001) for the splitting, $\Delta 2 \theta$, between 004 and 400/040 reflections in powder X-ray diffraction patterns. These refer to the right hand axis and have been scaled so as to overlap with data for $e_{\mathrm{t}}$. Solid lines are calculated variations of $e_{\mathrm{t}}$ and $e_{\mathrm{a}}$ at $295 \mathrm{~K}$ for a tricritical model of the cubic $\leftrightarrow$ tetragonal transition in the range CST0-CST92. Broken lines refer to a model (246 solution) in which the transition is tricritical in CST0, but becomes progressively more nearly second order with increasing $\mathrm{Sr}^{-}$content (M.A. Carpenter, unpublished data). (b) Predicted variations of single crystal elastic constants due to the $P m \overline{3} \mathrm{~m} \leftrightarrow I 4 / \mathrm{mcm}$ transition as a function of composition across the CST solid solution at $295 \mathrm{~K}$ (M.A. Carpenter, unpublished data). The value of $\lambda_{4}$ was set at $-0.131 \mathrm{GPa}$ for CST0-CST92 (tricritical model). (c) Comparison of observed (filled circles) and calculated (solid lines) bulk and shear moduli. The observed variations are from pulse-echo ultrasonic measurements on polycrystalline pellets (M.A. Carpenter, unpublished data). Calculated variations are averages of the Reuss and Voigt limits from the single crystal elastic constants shown in (b). Approximate composition limits for the $\mathrm{Pbcm}$ structure at room temperature are from Mishra et al. (2006). Data for $\mathrm{CaTiO}_{3}$ and $\mathrm{SrTiO}_{3}$ (open circles) are values taken from the literature (Bell and Rupprecht, 1963; Liebermann et al., 1977; Ishidate et al., 1988; Ledbetter et al., 1990; Fischer et al., 1993; Sinelnikov et al., 1998; Kung and Rigden, 1999; Lheureux et al., 1999; Webb et al., 1999). 
$T_{\mathrm{c} 2}$ replaced by $T_{\mathrm{c} 2 \mathrm{x}}$ to represent the values of $T_{\mathrm{c} 2}$ at each composition. Values of the strains are then given by Equations 5 and 6, and these are compared with experimental data from the literature in Figure 8a. Close agreement between the experimental data and the calculated strain variations suggests that the tricritical model might also yield robust predictions of single crystal elastic constants and bulk moduli. The latter are shown in Figures $8 \mathrm{~b}$ and $8 \mathrm{c}$, respectively.

Experimental data for individual elastic constants of intermediate members of the solid solution do not exist, but values of $K$ and $G$ have recently been determined from pulse-echo ultrasonic measurements on polycrystalline pellets of synthetic samples with 16 different compositions (M.A. Carpenter, unpublished data). These have been added to Figure $8 \mathrm{c}$ for comparison with the predicted variations. As with data for $\mathrm{SrTiO}_{3}$ as a function of temperature (Fig. 7a), scatter in the experimental data for $K$ is greater than the small anomaly which is predicted at the transition point. Also as for $\mathrm{SrTiO}_{3}$, the experimentally determined values of $G$ for tetragonal samples are significantly softer than the predicted values. This additional softening is at present believed to be due to anelastic effects associated with twin wall motion. The shear modulus of samples with the Pnma structure is significantly stiffer than it is for samples with the $I 4 / \mathrm{mcm}$ structure (Fig. 8c).

\section{ELASTIC ANOMALIES AS A FUNCTION OF PRESSURE}

The effect of pressure is introduced into Equation 1 by adding a term $P e_{\mathrm{a}}$, where $e_{\mathrm{a}}$ is the volume strain (Eqtn. 4). For a thermodynamically continuous $P m \overline{3} m \leftrightarrow I 4 / \mathrm{mcm}$ transition (second order, 246 or tricritical), the transition pressure, $P_{\mathrm{c}}$, at a given temperature, $T$, is then given by M.A. Carpenter (unpublished data) as

$$
P_{\mathrm{c}}=\frac{a_{2} \Theta_{\mathrm{s} 2} \frac{1}{3}\left(C_{11}^{\mathrm{o}}+2 C_{12}^{\mathrm{o}}\right)}{2 \lambda_{2}}\left(\operatorname{coth}\left(\frac{\Theta_{\mathrm{s} 2}}{T}\right)-\operatorname{coth}\left(\frac{\Theta_{\mathrm{s} 2}}{T_{\mathrm{c} 2 \mathrm{x}}}\right)\right) .
$$

From the known variation of $T_{\mathrm{c} 2}$ with composition, i.e., $T_{\mathrm{c} 2 \mathrm{x}}$, it is straightforward to predict $P_{\mathrm{c}}$ at room temperature for $\mathrm{Sr}$-rich compositions (Fig. 4b). A general expression for the evolution of $q_{4}$ becomes, for the case of a tricritical transition,

$$
q_{4}^{4}=\frac{2 \lambda_{2}}{\frac{1}{3}\left(C_{11}^{\mathrm{o}}+2 C_{12}^{\mathrm{o}}\right)\left(c_{2}+c_{2}^{\prime \prime}\right)}\left(P-P_{\mathrm{c}}\right) .
$$

Variations of individual elastic constants and bulk moduli due to the transition can then be calculated as before.
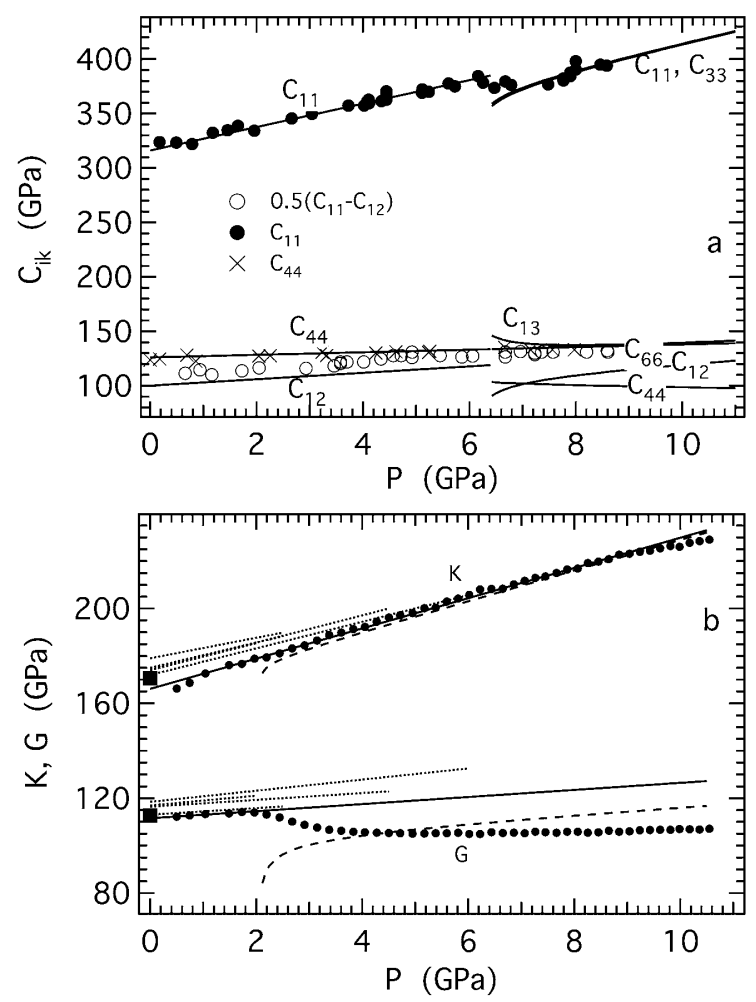

Figure 9. Elastic properties of CST perovskites at high pressures (from M.A. Carpenter, unpublished data). (a) Calculated variations (curves) and observed variations (data points, from Ishidate et al., 1988) of single crystal elastic constants for $\mathrm{SrTiO}_{3}$ as a function of pressure at room temperature. Straight lines are fits to $C_{11}, C_{12}$ and $C_{44}$ for cubic $\mathrm{SrTiO}_{3}$ given by Ishidate et al. (b) Comparison of observed (pulse-echo ultrasonic data, filled circles) and calculated variations of bulk and shear moduli as a function of pressure for CST95 at room temperature. Straight lines are fits to the data at low pressures for cubic states, while broken lines are the calculated variations of $K$ and $G$ for tetragonal CST95 $\left(\lambda_{4}=-0.11 \mathrm{GPa}, P_{\mathrm{c}}=2.11 \mathrm{GPa}\right)$, relative to the extrapolation of the fits to data for the cubic structure. Dotted lines represent trends of data from the literature for $\mathrm{SrTiO}_{3}$ (Beattie and Samara, 1971; Ishidate et al., 1988; Fischer et al., 1989, 1993; Lheureux et al., 1999).

A comparison of calculated and observed single crystal elastic constants for $\mathrm{SrTiO}_{3}$ is shown in Figure 9a. The single crystal data given by Ishidate et al. (1988) were collected using Brillouin spectroscopy. At pressures above $6.4 \mathrm{GPa}$ they were from within a single twin domain of the tetragonal structure (Ishidate et al., 1988). Neither of $C_{66}$ and $\frac{1}{2}\left(C_{11}-C_{12}\right)$ show any deflection through the transition point, as predicted by applying Equation 7 to Equation 1, while the data for $C_{11}$ agree closely with the calculated variations. A comparison of observed and calculated variations of the bulk and shear moduli for CST95 is given in Figure 9b. In this case, the data were obtained in-situ at high pressures using pulseecho ultrasonics and a polycrystalline sample. The calcu- 
lated variations are based on a solution for $q_{4}$ and $e_{\mathrm{t}}$ which is somewhat speculative in assuming that the thermodynamic character is intermediate between tricritical for CST92 and 246 for $\mathrm{SrTiO}_{3}$, however, $\left(\lambda_{4}=-0.11 \mathrm{GPa}\right)$. The true evolution of $q_{4}$ at this composition remains to be determined. (Heat capacity measurements of Gallardo et al. (2003) are consistent with second order character for the transition as a function of temperature in CST96, though their data for the intensities of superlattice reflections are not inconsistent with a 246 solution). Once again, there is little or no deflection of the bulk modulus at the transition point, due to only weak coupling between the order parameter and the volume strain. As with other ultrasonic measurements on twinned tetragonal crystals, the observed softening of the shear modulus differs significantly from the variation calculated on the basis of a standard strain/order parameter coupling model for the transition.

\section{ANELASTICITY AND THE INFLUENCE OF TRANSFORMATION TWIN WALLS}

Cubic $\leftrightarrow$ tetragonal transitions characteristically give rise to a microstructure of transformation twins. The spontaneous strain orientation for one twin is different from that of an adjacent twin, so movement of the wall separating them can change the total macroscopic strain of the crystal. If the twin wall moves in response to an applied shear stress, the total resulting strain can become substantially greater than would occur by simple lattice bending and the crystal will be anomalously soft (Schranz et al., 1999; Kityk et al., 2000a, 2000b; Binder and Knorr, 2001; Lemanov et al., 2002; Harrison and Redfern, 2002; Harrison et al., 2003, 2004a, 2004b, 2004c). This mechanism of softening involves some dissipation of energy and is anelastic in character. At high temperatures in tetragonal CST perovskites, the dissipation and softening is large, but below a temperature interval centered on $\sim 410 \mathrm{~K}$, both effects are substantially reduced (Harrison et al., 2003). Harrison et al. attributed this change to pinning of the twin walls by defects which are mobile at high temperatures but become immobile during cooling. Some anelastic softening occurs in $\mathrm{SrTiO}_{3}$ below the freezing temperature, however, as seen in published ultrasonic data obtained at $\mathrm{MHz}$ measuring frequencies (Figs. 6 and 7) and in Dynamical Mechanical Analysis (DMA) data collected at 1-350 Hz (Schranz et al., 1999; Kityk et al., $2000 \mathrm{a}, 2000 \mathrm{~b}$ ). Softening of the shear modulus of tetragonal CST beyond what is expected as a consequence of strain/order parameter coupling alone is also seen at room temperature in the pulse-echo ultrasonic data shown in Figure 8c. Evidence that this softening could be due to
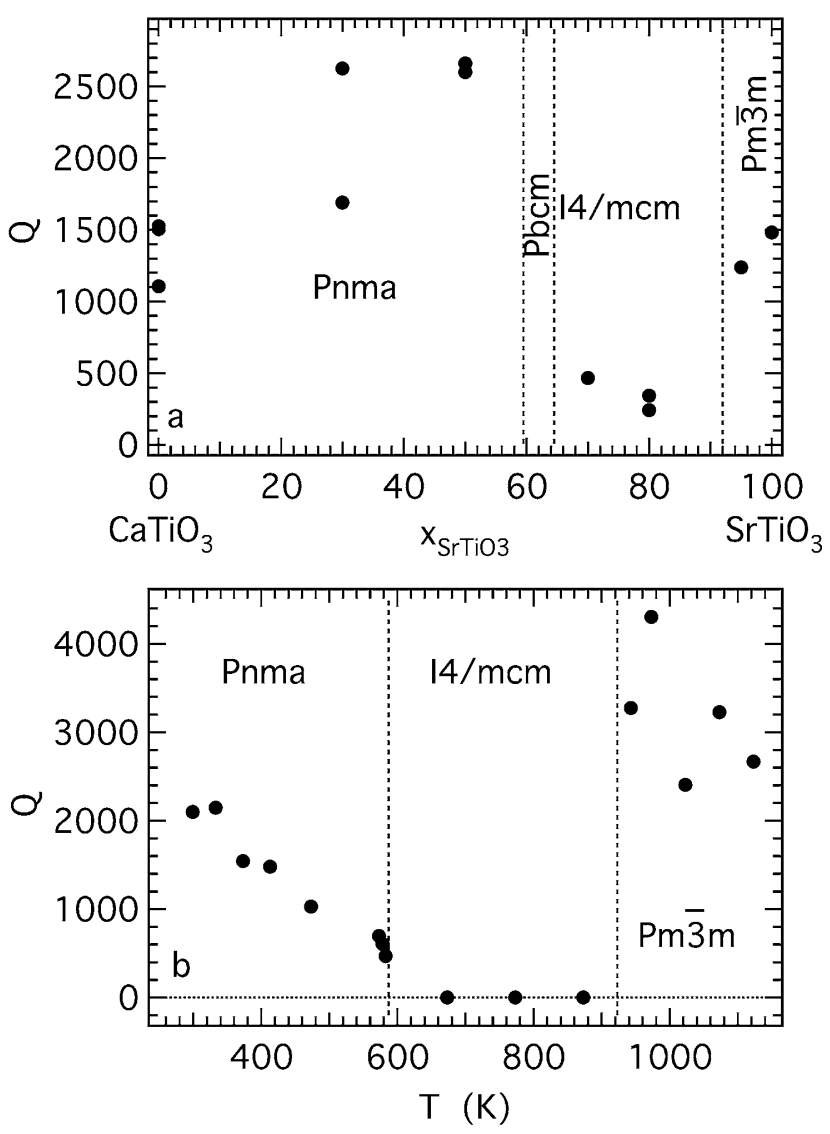

Figure 10. Attenuation in CST perovskites from Resonant Ultrasound Spectroscopy (J.W. Walsh, unpublished data). (a) Variation of the quality factor, $Q$, for polycrystalline samples of CST at room temperature. Tetragonal samples have significantly lower values of $Q$ than cubic or orthorhombic samples, consistent with the view that transformation twins are responsible for a degree of anelastic softening, even at room temperature and frequencies of hundreds of kHz. (b) Variation of $Q$ as a function of temperature for CST50. Transition temperatures delimiting the $14 / \mathrm{mcm}$ stability field were taken from Figure 4a. $Q$ has been assigned a value of zero for temperatures at which resonance peaks disappeared from the RUS spectra. The resonance peaks reappeared on heating into the stability field of the cubic structure and on cooling into the stability field of the orthorhombic structure. A high mobility of transformation twin walls at high temperatures is probably responsible for complete attenuation of the signal in tetragonal samples.

anelastic effects is provided by the marked attenuation observed in CST70 and CST80 by Resonant Ultrasound Spectroscopy (RUS) at room temperature (Fig. 10a). Figure 10 shows the variation of the quality factor, $Q$, as a function of composition from RUS measurements on polycrystalline samples with $\sim 5 \%$ porosity (J.W. Walsh, unpublished data). The quality factor is defined as $Q=(v /$ $\Delta v)$, where $v$ is the frequency of a strong resonance peak within the frequency range $0.4-1.4 \mathrm{MHz}$ and $\Delta v$ is the peak width at half height, and is related to the inverse of 

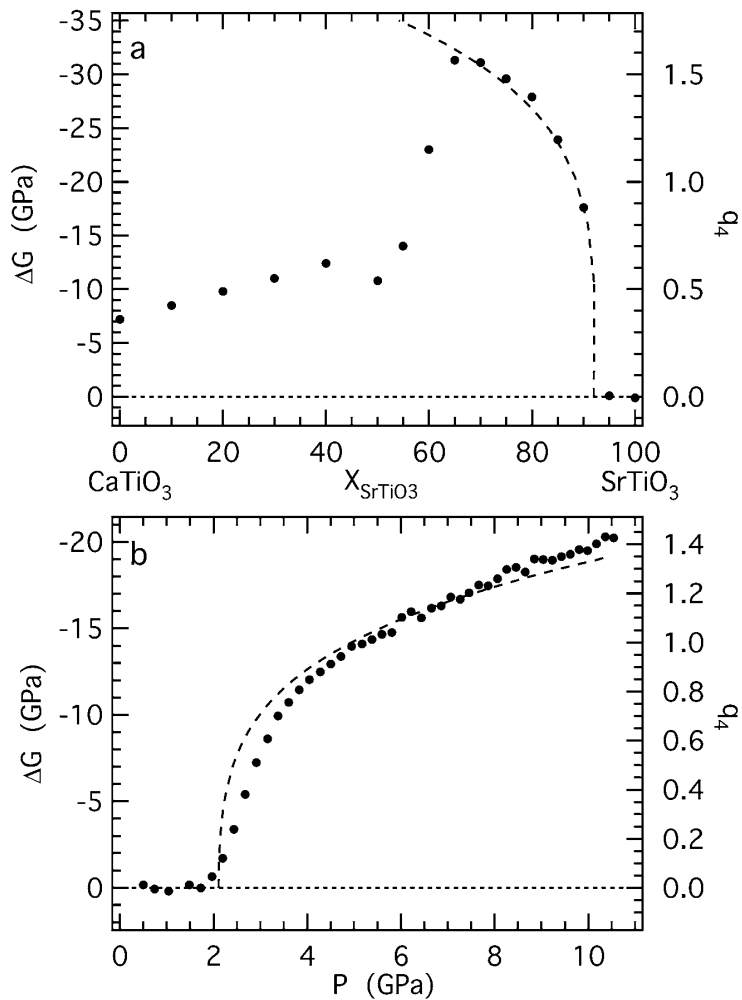

Figure 11. Change in shear modulus, $\Delta G$, as a function of composition and pressure at room temperature for CST perovskites, due to the change from cubic to tetragonal or orthorhombic symmetry (M.A. Carpenter, unpublished data). (a) $\Delta G$ was taken as the difference between the observed shear modulus values shown in Figure $8 \mathrm{c}$ and a constant value for cubic CST. In the compositional range of tetragonal CST, $\Delta G$ scales approximately with $q_{4}$ for the tricritical Landau model (broken line, right axis). (b) The difference in shear modulus between cubic (straight line in Fig. 9b) and tetragonal CST95 (filled circles in Fig.9b) as a function of pressure also scales approximately with $q_{4}$ from a Landau model (broken line, right axis).

the attenuation parameter, $\tan \delta$, from DMA experiments (Lee et al., 2000). Details of the dissipation mechanism at $\mathrm{MHz}$ frequencies are not known, but twin wall movements could be a more localised bulging between pinning points rather than the macroscopic displacements observed by Harrison et al. (2004a, 2004b, 2004c). At high temperatures resonance peaks in RUS spectra from polycrystalline tetragonal CST samples disappear, implying complete attenuation $(Q \approx 0)$. They reappear when the structure becomes cubic at higher temperature or orthorhombic at lower temperatures (Fig. 10b), and the influence of high twin wall mobilities in tetragonal CST is again believed to be involved.

Elastic compliances for different contributions to the elastic properties of a crystal are additive. Thus the compliance, $S$, of a twinned tetragonal crystal is expected to be given by

$$
S=S_{\text {para }}+\Delta S_{\mathrm{L}}+\Delta S_{\mathrm{W}}
$$

where $S_{\text {para }}$ is the compliance of the high symmetry form, $\Delta S_{\mathrm{L}}$ is the change in compliance described by Landau theory and $\Delta S_{\mathrm{W}}$ is the change in compliance due to movements of the twin walls (Schranz et al., 1999; Kityk et al., 2000a, 2000b). Given that the total strain accompanying twin wall movement scales with $e_{\mathrm{t}}$ and, hence, with $q_{4}^{2}$, the simplest model has $\Delta S_{\mathrm{W}} \propto q_{4}^{2}$. This provides an effective description of softening in single crystals of $\mathrm{SrTiO}_{3}$ (Schranz et al., 1999; Kityk et al., 2000a, 2000b). A different empirical relationship appears to provide a better description of the softening of $G$ for polycrystalline samples of tetragonal CST, however. This has the change in shear modulus, $\Delta G$, due to the cubic $\leftrightarrow$ tetragonal transition varying according to $\Delta G \propto q_{4}$ as a function of composition at room temperature and as a function of pressure at room temperature for CST95 (Fig. 11). Such an unexpected approximation must arise, at least in part, as a consequence of averaging over all twin wall orientations in a polycrystalline material. Some of the walls will be oriented with respect to an applied shear stress so as to experience the maximum possible displacement. Some will be oriented in such a way that they are not subject to any resolved stress, while most will have orientations intermediate between these limits and therefore be responsible for a range of intermediate strains, as shown in Figure 9 of Harrison et al. (2003).

\section{THE I4/mcm $\leftrightarrow$ Pnma TRANSITION}

It has not yet been possible to extend the parameterisation of Equation 1 to all the terms which describe tilting transitions of CST that are associated with the M point of the Brillouin zone $\left(T_{\mathrm{c} 1}, a_{1}, b_{1}, \ldots, \lambda_{1}, \lambda_{3}, \ldots\right.$, etc.). Even without the quantitative information that such a parameterisation would provide, however, it is possible to predict the form of elastic anomalies which might accompany the $I 4 / \mathrm{mcm} \leftrightarrow$ Pnma transition. From expressions for the Voigt limits for changes in $K$ and $G$ due to a $P m \overline{3} m \leftrightarrow$ Pnma transition (M.A. Carpenter, unpublished data),

$$
\begin{aligned}
K_{\mathrm{V}} & =\frac{1}{3}\left(C_{11}^{\mathrm{o}}+2 C_{12}^{\mathrm{o}}\right)-4 \lambda_{1}^{2} \chi_{2} q_{2}^{2}-8 \lambda_{2}^{2} \chi_{4} q_{4}^{2} \\
G_{\mathrm{V}} & =\frac{1}{5}\left(C_{11}^{\mathrm{o}}-C_{12}^{\mathrm{o}}+3 C_{44}^{\mathrm{o}}\right)-\frac{2}{5}\left(8 \lambda_{3}^{2} \chi_{2}+3 \lambda_{6}+2 \lambda_{7}\right) q_{2}^{2} \\
& -\frac{2}{5}\left(16 \lambda_{4}^{2} \chi_{4}+\lambda_{5}^{2} \chi_{4}+\lambda_{5}^{2} \chi_{5}\right) q_{4}^{2},
\end{aligned}
$$

the Pnma structure in a sequence $P m \overline{3} m \leftrightarrow I 4 / m c m \leftrightarrow$ Pnma would be expected to be elastically softer than the 


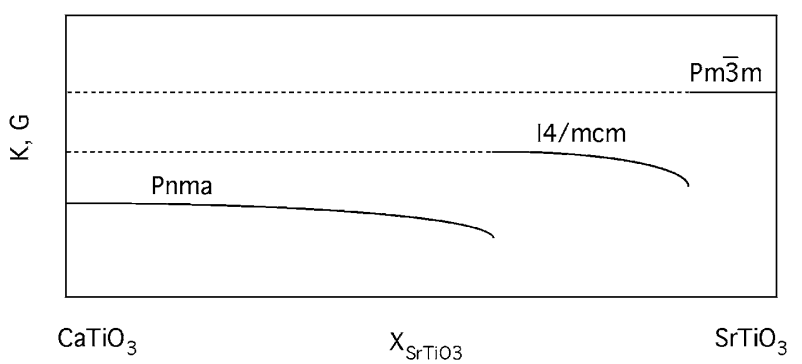

Figure 12. Schematic representation of relative changes in bulk modulus and shear modulus through the sequence of transitions $P m \overline{3} m \leftrightarrow I 4 / m c m \leftrightarrow P n m a$ in perovskites. On the basis of Landau expansions for CST (M.A. Carpenter, unpublished data) and excluding the effects of microstructure, each transition is expected to give rise to discontinuous softening of both $K$ and $G$ with falling symmetry (see Equations 12, 13, 17, 18). The amount of curvature just below the transition point depends on how close the evolution of the equilibrium order parameter is to tricritical. Decreasing temperature and increasing pressure should give a sequence which is analogous to increasing $\mathrm{CaTiO}_{3}$ content.

$I 4 / \mathrm{mcm}$ structure (compare Equations 12 and 13 with Equations 17 and 18). This is because, in the absence of unusual direct coupling effects between $q_{2}$ and $q_{4}$, the value of $q_{4}^{2}$ in the Pnma structure will be close to half its value in the $I 4 / \mathrm{mcm}$ structure. If the M point instability is also close to tricritical and the coupling coefficients $\lambda_{6}$ and $\lambda_{7}$ are small, the bulk and shear moduli would be expected to evolve in the manner illustrated in Figure 12 for pressure, temperature or composition as the external variable. That this pattern is not observed (e.g., see Fig. 8c) might be understood in terms of the twin wall related anelasticity of $I 4 / \mathrm{mcm}$ samples. Harrison et al. (2003) found that anelastic contributions to the overall elastic behaviour of CST effectively disappear as soon as $14 / \mathrm{mcm}$ symmetry is broken. This is confirmed for frequencies of hundreds of $\mathrm{kHz}$ by the increase of $Q$ observed by RUS for Pnma samples, relative to $I 4 / \mathrm{mcm}$ samples (Fig. 10). The reason for such an abrupt change in properties of the twin walls is not understood, but they could simply become immobile (Harrison et al., 2003). Alternatively, the change in tetragonal strain which accompanies the $I 4 / \mathrm{mcm} \leftrightarrow P n m a$ transition could have a bearing. In the Pnma structure the tetragonal strain defined with respect to the crystallographic $\mathrm{y}$-axis, $e_{\mathrm{tx}}$, varies with the $\mathrm{R}$ point and $\mathrm{M}$ point order parameter components according to (Carpenter et al., 2001)

$$
e_{\mathrm{tx}}=-\frac{\left(2 \lambda_{3} q_{2}^{2}-\lambda_{4} q_{4}^{2}\right)}{\frac{1}{2}\left(C_{11}^{\mathrm{o}}-C_{12}^{\mathrm{o}}\right)} .
$$

This is close to zero at intermediate compositions in the
CST solid solution (Carpenter et al., 2001), implying that $\lambda_{3}$ and $\lambda_{4}$ have the same sign. The net change in strain due to displacement of a twin wall will also be close to zero, therefore, and the elasticity of the bulk crystal will not be affected. Whatever the mechanism might be, the result is that Pnma structures are stiffer than twinned $14 / \mathrm{mcm}$ structures (Harrison et al., 2003; M.A.Carpenter, unpublished data; Fig. 8c).

\section{IMPLICATIONS FOR THE ELASTIC BEHAVIOUR OF SILICATE PEROVSKITES}

There seems to be a current consensus that $(\mathrm{Mg}, \mathrm{Fe}) \mathrm{SiO}_{3}$ perovskite has Pnma symmetry at all pressures and temperatures where it is present in the mantle (Wentzcovitch et al., 1993; Stixrude and Cohen, 1993; Fiquet et al., 1998; Ono et al., 2004a; and references therein). So long as these conditions are substantially away from the tetragonal $\leftrightarrow$ orthorhombic transition point, the amount of softening due to the transition itself is expected to be more or less independent of $q_{2}$ and $q_{4}$. In other words, changes in octahedral tilting with changing $P, T$ or composition in the Pnma field will not contribute any additional softening through strain/order parameter coupling effects. If the twin walls are immobile, additional anelastic softening associated with a transformation twin microstructure can be ruled out.

For $\mathrm{CaSiO}_{3}$, the most recent experimental and computational results appear to be favouring a view that the tetragonal $(I 4 / \mathrm{mcm})$ structure is stable at ambient conditions and that the cubic $(P m \overline{3} m)$ structure could be stable at mantle conditions (Ono et al., 2004b; Kurashina et al., 2004; Caracas et al., 2005; Jung and Oganov, 2005). Addition of Al substantially stabilises the orthorhombic structure, however (Kurashina et al., 2004). If a cubic $\leftrightarrow$ tetragonal transition occurred in $\mathrm{CaSiO}_{3}$ within the mantle, it should give rise to a marked anomaly in the shear modulus, as would a tetragonal $\leftrightarrow$ orthorhombic transition. Almost inevitably, tetragonal $\mathrm{CaSiO}_{3}$ would retain some twinning microstructure, with the implication that the shear modulus would be significantly softer than for either of the cubic or orthorhombic phases and that substantial attenuation of seismic waves would also occur. For pressures and temperatures above the domain wall freezing interval, the amount of softening and attenuation would be expected to be more or less independent of temperature (Harrison and Redfern, 2002; Harrison et al., 2003). For pressures and temperatures below this interval similar effects might be expected, though to a lesser extent and with a strong correlation with the degree of octahedral tilting. If coupling between octahedral tilting and the volume strain is as weak in silicate perovskites as it is 
in CST perovskite, any anomalies in the bulk modulus due to tilting phase transitions will be small.

\section{ACKNOWLEDGMENTS}

This work was funded by grants from the Natural Environment Research Council (NER/A/S/2000/01055, NE/B505738/1), which are gratefully acknowledged. The ultrasonic experiments on spodumene and CST perovskites were performed in the Stony Brook High Pressure Laboratory, with partial support of NSF grants to B. Li (EAR0003340) and to R.C. Liebermann (EAR02-29704).

\section{REFERENCES}

Agee, C.B. (1998) Phase transformations and seismic structure in the upper mantle and transition zone. In Ultrahigh-Pressure Mineralogy: Physics and Chemistry of the Earth's Deep Interior (Hemley, R.J. Ed.). Reviews in Mineralogy, Mineralogical Society of America, Chantilly, 37, 165-203.

Angel, R.J., Zhao, J. and Ross, N.L. (2005) General rules for predicting phase transitions in perovskites due to octahedral tilting. Physical Review Letters, 95, 025503.

Beattie, A.G. and Samara, G.A. (1971) Pressure dependence of the elastic constants of $\mathrm{SrTiO}_{3}$. Journal of Applied Physics, 42, 2376-2381.

Bell, R.O. and Rupprecht, G. (1963) Elastic constants of strontium titanate. Physical Review, 129, 90-94.

Binder, A. and Knorr, K. (2001) Shear elasticity and ferroelastic hysteresis of the low-temperature phase of $\mathrm{SrTiO}_{3}$. Physical Review B, 63, 094106.

Boffa Ballaran, T. and Carpenter, M.A. (2003) Line broadening and enthalpy: some empirical calibrations of solid solution behaviour from IR spectra. Phase Transitions, 76, 137-154.

Bonello, B., Fischer, M. and Zarembowitch, A. (1989a) Acoustical measurements in solids under very high pressure. Ultrasonics, 27, 343-348.

Bonello, B., Fischer, M. and Polian, A. (1989b) Phase transition and sound velocity measurements in very small solid samples under high pressure by Brillouin scattering. Journal of the Acoustical Society of America, 86, 2257-2260.

Caracas, R., Wentzcovitch, R., Price, G.D. and Brodholt, J. (2005) $\mathrm{CaSiO}_{3}$ perovskite at lower mantle pressures. Geophysical Research Letters, 32, L06306 doi:10.1029/2004GL022144.

Carpenter, M.A. (1988) Thermochemistry of aluminium/silicon ordering in feldspar minerals. In Physical Properties and Thermodynamic Behaviour of Minerals (Salje, E.K.H. Ed.). NATO ASI Series C, Reidel, Dordrecht, 225, 265-323.

Carpenter, M.A. (1992) Thermodynamics of phase transitions in minerals: a macroscopic approach. In The Stability of Minerals (Price, G.D. and Ross, N.L. Eds.). Chapman and Hall, London, 172-215.

Carpenter, M.A. (2000) Strain and elasticity at structural phase transitions in minerals. In Transformation Processes in Minerals (Redfern, S.A.T. and Carpenter, M.A. Eds.). Reviews in Mineralogy and Geochemistry, Mineralogical Society of America, Chantilly, 39, 35-64.

Carpenter, M.A. (2002) Microscopic strain, macroscopic strain and the thermodynamics of phase transitions in minerals.
European Mineralogical Union Notes in Mineralogy, 4, 311346.

Carpenter, M.A. (2006) Elastic properties of minerals and the influence of phase transitions. American Mineralogist, 91, 229-246.

Carpenter, M.A. and Salje, E.K.H. (1998) Elastic anomalies in minerals due to structural phase transitions. European Journal of Mineralogy, 10, 693-812.

Carpenter, M.A., Salje, E.K.H. and Graeme-Barber, A. (1998a) Spontaneous strain as a determinant of thermodynamic properties for phase transitions in minerals. European Journal of Mineralogy, 10, 621-691.

Carpenter, Salje, E.K.H., Graeme-Barber, A., Wruck, B., Dove, M.T. and Knight, K.S. (1998b) Calibration of excess thermodynamic properties and elastic constant variations associated with the $\alpha \leftrightarrow \beta$ phase transition in quartz. American Mineralogist, 83, 2-22.

Carpenter, M.A., Hemley, R.J. and Mao, H.-K. (2000) High-pressure elasticity of stishovite and the $P 4_{2} / m n m \leftrightarrow P m n n$ phase transition. Journal of Geophysical Research, 105, 1080710816.

Carpenter, M.A. and Boffa Ballaran, T. (2001) The influence of elastic strain heterogeneities in silicate solid solutions. European Mineralogical Union Notes in Mineralogy, 3, 155178.

Carpenter, M.A., Becerro, A.I., and Seifert, F. (2001) Strain analysis of phase transitions in $(\mathrm{Ca}, \mathrm{Sr}) \mathrm{TiO}_{3}$ perovskites. American Mineralogist, 86, 348-363.

Conti, S. and Salje, E.K.H. (2001) Surface structure of ferroelastic domain walls: a continuum elasticity approach. Journal of Physics: Condensed Matter, 13, L847-L854.

Cordier, P., Mainprice, D. and Mosenfelder, J.L. (2004) Mechanical instability near the stishovite $-\mathrm{CaCl}_{2}$ phase transition: implications for crystal preferred orientations and seismic properties. European Journal of Mineralogy, 16, 387-399.

Fiquet, G., Andrault, D., Dewaele, A., Charpin, T., Kunz, M. and Haüsermann, D. (1998) P-V-T equation of state of $\mathrm{MgSiO}_{3}$ perovskite. Physics of the Earth and Planetary Interiors, 105, 21-31.

Fischer, M., Bonnello, B., Polian, A. and Léger, J.-M. (1989) Elasticity of $\mathrm{SrTiO}_{3}$ perovskite under high pressure. In Perovskite: a structure of great interest to Geophysics and Materials Science (Navrotsky, A. and Weidner, D.J. Eds.,). Geophysical Monograph, 45, 125-130.

Fischer, G.J., Wang, Z. and Karato, S.-I. (1993) Elasticity of $\mathrm{CaTiO}_{3}, \mathrm{SrTiO}_{3}$ and $\mathrm{BaTiO}_{3}$ perovskites up to $3.0 \mathrm{GPa}$ : the effect of crystallographic structure. Physics and Chemistry of Minerals, 20, 97-103.

Fossheim, K. and Berre, B. (1972) Ultrasonic propagation, stress effects, and interaction parameters at the displacive transition in $\mathrm{SrTiO}_{3}$. Physical Review B, 5, 3292-3308.

Gallardo, M.C., Becerro, A.I., Romero, F.J., del Cerro, J., Seifert, F. and Redfern, S.A.T. (2003) Cubic-tetragonal phase transition in $\mathrm{Ca}_{0.04} \mathrm{Sr}_{0.96} \mathrm{TiO}_{3}$ : a combined specific heat and neutron diffraction study. Journal of Physics: Condensed Matter, 15, 91100.

Glazer, A.M. (1972) The classification of tilted octahedra in perovskites. Acta Crystallographica, B28, 3384-3392.

Glazer, A.M. (1975) Simple ways of determining perovskite structures. Acta Crystallographica, A31, 756-762.

Grzechnik, A., Wolf, G.H. and McMillan, P.F. (1997) Raman scattering study of $\mathrm{SrTiO}_{3}$ at high pressure. Journal of Raman 
Spectroscopy, 28, 885-889.

Harrison, R.J. and Redfern, S.A.T. (2002) The influence of transformation twins on the seismic-frequency elastic and anelastic properties of perovskite: dynamical mechanical analysis of single crystal $\mathrm{LaAlO}_{3}$. Physics of the Earth and Planetary Interiors, 134, 253-272.

Harrison, R.J., Redfern, S.A.T. and Street, J. (2003) The effect of transformation twins on the seismic-frequency mechanical properties of polycrystalline $\mathrm{Ca}_{1-\mathrm{x}} \mathrm{Sr}_{\mathrm{x}} \mathrm{TiO}_{3}$ perovskite. American Mineralogist, 88, 574-582.

Harrison, R.J., Redfern, S.A.T. and Salje, E.K.H. (2004a) Dynamical excitation and anelastic relaxation of ferroelastic domain walls in $\mathrm{LaAlO}_{3}$. Physical Review B, 69, 144101.

Harrison, R.J., Redfern, S.A.T. and Bismayer, U. (2004b) Seismic-frequency attenuation at first-order phase transitions: dynamical mechanical analysis of pure and $\mathrm{Ca}$-doped lead orthophosphate. Mineralogical Magazine, 68, 839-852.

Harrison, R.J., Redfern, S.A.T., Buckley, A. and Salje, E.K.H. (2004c) Application of real-time, stroboscopic X-ray diffraction with dynamical mechanical analysis to characterize the motion of ferroelastic domain walls. Journal of Applied Physics, 95, 1706-1717.

Hayward, S.A. and Salje, E.K.H. (1999) Cubic-tetragonal phase transition in $\mathrm{SrTiO}_{3}$ revisited: Landau theory and transition mechanism. Phase Transitions, 68, 501-522.

Hemley, R.J., Shu, J.F., Carpenter, M.A., Hu, J., Mao, H.-K. and Kingma, K.J. (2000) Strain/order parameter coupling in the ferroelastic transition in dense $\mathrm{SiO}_{2}$. Solid State Communications, 114, 527-532.

Hill, R. (1952) The elastic behaviour of a crystalline aggregate. Proceedings of the Physical Society of London A, 65, 349354.

Hirose, K., Takafuji, N., Sata, N. and Ohishi, Y. (2005) Phase transition and density of subducted MORB crust in the lower mantle. Earth and Planetary Science Letters, 237, 239-251.

Howard, C.J., and Stokes, H.T. (1998) Group-theoretical analysis of octahedral tilting in perovskite. Acta Crystallographica B, 54, 782-789.

Howard, C.J., Withers, R.L., Zhang, Z., Osaka, K., Kato, K. and Takata, M. (2005) Space-group symmetry for the perovskite $\mathrm{Ca}_{0.3} \mathrm{Sr}_{0.7} \mathrm{TiO}_{3}$. Journal of Physics: Condensed Matter, 17, L459-L465.

Ishidate, T. and Isonuma, T. (1992) Phase transition of $\mathrm{SrTiO}_{3}$ under high pressure. Ferroelectrics, 137, 45-52.

Ishidate, T., Sasaki, S. and Inoue, K. (1988) Brillouin scattering of $\mathrm{SrTiO}_{3}$ under high pressure. High Pressure Research, 1, 5365.

Jung, D.Y. and Oganov, A.R. (2005) Ab initio study of the highpressure behavior of $\mathrm{CaSiO}_{3}$ perovskite. Physics and Chemistry of Minerals, $32,146-153$.

Kern, H. (1979) Effect of high-low quartz transition on compressional and shear wave velocities in rocks under high pressure. Physics and Chemistry of Minerals, 4, 161-171.

Kern, H. (1982) Elastic-wave velocity in crustal and mantle rocks at high pressure and temperature: the role of the high-low quartz transition and of dehydration reactions. Physics of the Earth and Planetary Interiors, 29, 12-23.

Kityk, A.V., Schranz, W., Sondergeld, P., Havlik, D., Salje, E.K.H. and Scott, J.F. (2000a) Low-frequency superelasticity and nonlinear elastic behavior of $\mathrm{SrTiO}_{3}$ crystals. Physical Review B, 61, 946-956.

Kityk, A.V., Schranz, W., Sondergeld, P., Havlik, D., Salje, E.K.H. and Scott, J.F. (2000b) Nonlinear elastic behaviour of $\mathrm{SrTiO}_{3}$ crystals in the quantum paraelectric regime. Europhysics Letters, 50, 41-47.

Kung, J. and Rigden, S. (1999) Oxide perovskites: pressure derivatives of the bulk and shear moduli. Physics and Chemistry of Minerals, 26, 234-241.

Kurashina, T., Hirose, K., Ono, S., Sata, N. and Ohishi, Y. (2004) Phase transition in $\mathrm{Al}$-bearing $\mathrm{CaSiO}_{3}$ perovskite: implications for seismic discontinuities in the lower mantle. Physics of the Earth and Planetary Interiors, 145, 67-74.

Ledbetter, H., Lei, M. and Sudook, K. (1990) Elastic constants, Debye temperatures, and electron-phonon parameters of superconducting cuprates and related oxides. Phase Transitions, 23, 61-70.

Lee, T., Lakes, R.S. and Lal, A. (2000) Resonant ultrasound spectroscopy for measurement of mechanical damping: comparison with broadband viscoelastic spectroscopy. Review of Scientific Instruments, 71, 2855-2861.

Lei, M. (1991) Oxides and oxide superconductors: elastic and related properties. Ph.D. dissertation, University of Colorado at Boulder.

Lemanov, V.V. (1997) Phase transitions in $\mathrm{SrTiO}_{3}$-based solid solutions. Physics of the Solid State, 39, 1468-1473.

Lemanov, V.V., Gridnev, S.A. and Ukhin, E.V. (2002) Low-frequency elastic properties, domain dynamics, and spontaneous twisting of $\mathrm{SrTiO}_{3}$ near the ferroelastic phase transition. Physics of the Solid State, 44, 1156-1165.

Lheureux, D., Fischer, M., Polian, A., Itié, J.P., Gauthier, M. and Syfosse, G. (1999) Elastic properties of $\mathrm{SrTiO}_{3}$ under extreme conditions: a new high pressure ultrasonic set-up. 1999 IEEE Ultrasonics Symposium, 533-536.

Li, B.S., Rigden, S.M. and Liebermann, R.C. (1996) Elasticity of stishovite at high pressure. Physics of the Earth and Planetary Interiors, 96, 113-127.

Liebermann, R.C., Jones, L.E.A. and Ringwood, A.E. (1977) Elasticity of aluminate, titanate, stannate and germanate compounds with the perovskite structure. Physics of the Earth and Planetary Interiors, 14, 165-178.

Marini, L. and Manzella, A. (2005) Possible seismic signature of the $\alpha-\beta$ quartz transition in the lithosphere of southern Tuscany (Italy). Journal of Volcanology and Geothermal Research, 148, 81-97.

Mechie, J., Sobolev, S.V., Ratschbacher, L., Babeyko, A.Y., Bock, B., Jones, A.G., Nelson, K.D., Solon, K.D., Brown, L.D. and Zhao, W. (2004) Precise temperature estimation in the Tibetan crust from seismic detection of the $\alpha-\beta$ quartz transition. Geology, 32, 601-604.

Mishra, S.K., Ranjan, R., Pandey, D. and Stokes, H.T. (2006) Resolving the controversies about the 'nearly cubic' and other phases of $\mathrm{Sr}_{1-x} \mathrm{Ca}_{x} \mathrm{TiO}_{3}(0 \leq x \leq 1)$ : I. Room temperature structures. Journal of Physics: Condensed Matter, 18, 18851898.

Ono, S., Kikegawa, T. and lizuka, T. (2004a) The equation of state of orthorhombic perovskite in a peridotitic mantle composition to $80 \mathrm{GPa}$ : implications for chemical composition of the lower mantle. Physics of the Earth and Planetary Interiors, 145, 9-17.

Ono, S., Ohishi, Y. and Mibe, K. (2004b) Phase transition of Caperovskite and stability of $\mathrm{Al}$-bearing $\mathrm{Mg}$-perovskite in the lower mantle. American Mineralogist, 89, 1480-1485.

Qin, S., Becerro, A.I., Seifert, F., Gottsmann, J. and Jiang, J. (2000) Phase transitions in $\mathrm{Ca}_{1-\mathrm{x}} \mathrm{Sr}_{\mathrm{x}} \mathrm{TiO}_{3}$ perovskites: effects of com- 
position and temperature. Journal of Materials Chemistry, 10, 1609-1615.

Ranjan, R. and Pandey, D. (2001) Antiferroelectric phase transition in $\left(\mathrm{Sr}_{1-\mathrm{x}} \mathrm{Ca}_{\mathrm{x}}\right) \mathrm{TiO}_{3}(0.12<\mathrm{x} \leq 0.40)$ : I. Dielectric studies. Journal of Physics: Condensed Matter, 13, 4239-4249.

Ranjan, R., Pandey, D., Schuddinck, W., Richard, O., De Meulenaere, P., Van Landuyt, J. and Van Tendeloo, G. (2001) Evolution of crystallographic phases in $\left(\mathrm{Sr}_{1-\mathrm{x}} \mathrm{Ca}_{\mathrm{x}}\right) \mathrm{TiO}_{3}$ with composition (x). Journal of Solid State Chemistry, 162, 2028.

Redfern, S.A.T. (2000) Order-disorder phase transitions. In Transformation Processes in Minerals (Redfern, S.A.T. and Carpenter, M.A. Eds.). Reviews in Mineralogy and Geochemistry, Mineralogical Society of America, Chantilly, 39, 105133.

Rehwald, W. (1970) Low temperature elastic moduli of strontium titanate. Solid State Communications, 8, 1483-1485.

Rehwald, W. (1971) Ultrasonic properties of strontium titanate at the $105^{\circ} \mathrm{K}$ transition. Physik der Kondensierten Materie, 14, 21-36.

Salje, E.K.H. (1991) Crystallography and structural phase transitions, an introduction. Acta Crystallographica, A47, 453-469.

Salje, E.K.H. (1992) Application of Landau theory for the analysis of phase transitions in minerals. Physics Reports, 215, 49-99.

Salje, E.K.H., Gallardo, M.C., Jiménez, J., Romero, F.J. and del Cerro, J. (1998) The cubic-tetragonal phase transition in strontium titanate: excess specific heat measurements and evidence for a near-tricritical, mean field type transition mechanism. Journal of Physics: Condensed Matter, 10, 55355543.

Salje, E.K.H., Hayward, S.A. and Lee, W.T. (2005) Ferroelastic phase transitions: structure and microstructure. Acta Crystallographica A, 61, 3-18.

Schranz, W., Sondergeld, P., Kityk, A.V. and Salje, E.K.H. (1999) Elastic properties of $\mathrm{SrTiO}_{3}$ crystals at ultralow frequencies. Phase Transitions, 69, 61-76.

Sinelnikov, Y.D., Chen, G. and Liebermann, R.C. (1998) Elasticity of $\mathrm{CaTiO}_{3}-\mathrm{CaSiO}_{3}$ perovskites. Physics and Chemistry of Minerals, 25, 515-521.

Slonczewski, J.C. and Thomas, H. (1970) Interaction of elastic strain with the structural transition of strontium titanate. Phy- sical Review B, 1, 3599-3608.

Sondergeld, P., Li, B., Schreuer, J. and Carpenter, M.A. (2006) Discontinuous evolution of single crystal elastic constants as a function of pressure through the $C 2 / c \leftrightarrow P 2_{1} / c$ phase transition in spodumene, $\mathrm{LiAlSi}_{2} \mathrm{O}_{6}$. Journal of Geophysical Research, in press.

Stixrude, L. and Cohen, R.E. (1993) Stability of orthorhombic $\mathrm{MgSiO}_{3}$ perovskite in the earth's lower mantle. Nature, 364, 613-615.

Tarantino, S.C., Carpenter, M.A. and Domeneghetti, M.C. (2003) Strain and local heterogeneity in the forsterite-fayalite solid solution. Physics and Chemistry of Minerals, 30, 495-502.

Van Westrenen, W., Blundy, J. and Wood, B. (1999) Crystalchemical controls on trace element partitioning between garnet and anhydrous silicate melt. American Mineralogist, 84, 838-847.

Watt, J.P. (1979) Hashin-Shtrikman bounds on the effective elastic moduli of polycrystals with orthorhombic symmetry. Journal of Applied Physics, 50, 6290-6295.

Watt, J.P. and Peselnik. L. (1980) Clarification of the HashinShtrikman bounds on the effective elastic moduli of polycrystals with hexagonal, trigonal, and tetragonal symmetries. Journal of Applied Physics, 51, 1525-1531.

Webb, S., Jackson, I. and Fitz Gerald, J. (1999) Viscoelasticity of the titanate perovskites $\mathrm{CaTiO}_{3}$ and $\mathrm{SrTiO}_{3}$ at high temperatures. Physics of the Earth and Planetary Interiors, 115, 259291.

Wentzcovitch, R.M., Martins, J.L., and Price, G.D. (1993) Ab initio molecular dynamics with variable cell shape: application to $\mathrm{MgSiO}_{3}$. Physical Review Letters, 70, 3947-3950.

Woodward, P.M. (1997) Octahedral tilting in perovskites. 1. Geometrical considerations. Acta Crystallographica, B53, $32-43$.

Yamanaka, T., Hirai, N. and Komatsu, Y. (2002) Structure change of $\mathrm{Ca}_{1-\mathrm{x}} \mathrm{Sr}_{\mathrm{x}} \mathrm{TiO}_{3}$ perovskite with composition and pressure. American Mineralogist, 87, 1183-1189.

Manuscript received February 21, 2006

Manuscript accepted April 21, 2006

Manuscript handled by Kiyoshi Fujino 